\title{
Semiclassical measures on hyperbolic surfaces have full support
}

\author{
Semyon Dyatlov \\ Massachusetts Institute of Technology \\ Cambridge, MA, U.S.A. \\ and \\ University of California, Berkeley \\ Berkeley, CA, U.S.A.
}

\author{
by
}

\section{Introduction}

Let $(M, g)$ be a compact (connected) hyperbolic surface, that is a Riemannian surface of constant curvature -1 . Denote by $\Delta$ the (non-positive) Laplace-Beltrami operator. We fix a semiclassical quantization procedure $\mathrm{Op}_{h}$ (see §2.2) which maps any function $a \in C_{0}^{\infty}\left(T^{*} M\right)$ to a family of operators $\mathrm{Op}_{h}(a): L^{2}(M) \rightarrow L^{2}(M)$ depending on the semiclassical parameter $h>0$. Assume that $u_{j}$ is a sequence of eigenfunctions of $-\Delta$ with eigenvalues $h_{j}^{-2} \rightarrow \infty$ :

$$
\left(-h_{j}^{2} \Delta-I\right) u_{j}=0, \quad\left\|u_{j}\right\|_{L^{2}}=1, \quad h_{j}>0, \quad h_{j} \rightarrow 0 \text { as } j \rightarrow \infty .
$$

We say that $u_{j}$ converge semiclassically to some probability measure $\mu$ on $T^{*} M$ if

$$
\left\langle\mathrm{Op}_{h_{j}}(a) u_{j}, u_{j}\right\rangle_{L^{2}} \rightarrow \int_{T^{*} M} a d \mu \text { as } j \rightarrow \infty \quad \text { for all } a \in C_{0}^{\infty}\left(T^{*} M\right) .
$$

We say that $\mu$ is a semiclassical defect measure (or in short, semiclassical measure) if $\mu$ is the semiclassical limit of some sequence of eigenfunctions. It is well known (see for instance [Zw1, $§ 5.1$ and $\S 5.2]$ ) that each semiclassical defect measure is supported on the cosphere bundle $S^{*} M \subset T^{*} M$ and it is invariant under the geodesic flow $\varphi_{t}: S^{*} M \rightarrow S^{*} M$. However, not every invariant measure can be a semiclassical defect measure as follows from our first result.

TheOREM 1. Let $\mu$ be a semiclassical defect measure. Then, $\operatorname{supp} \mu=S^{*} M$, that is for every non-empty open set $\mathcal{U} \subset S^{*} M$ we have $\mu(\mathcal{U})>0$. 
If $a \in C^{\infty}(M)$ depends only on $x$, then $\mathrm{Op}_{h}(a)$ is the multiplication operator by $a$. Therefore, Theorem 1 implies that the support of any weak limit of the measures $\left|u_{j}\right|^{2} d \mathrm{vol}_{g}$ (often called quantum limit) is equal to $M$.

The quantum ergodicity theorem of Shnirelman [Sh], Zelditch [Ze1], and Colin de Verdière [CdV] (see also Helffer-Martinez-Robert [HMR] and Zelditch-Zworski [ZZ] for more general versions) implies that there is a density-1 sequence of eigenvalues of $\Delta$ such that the corresponding eigenfunctions converge weakly to the Liouville measure $\mu_{L}$. The quantum unique ergodicity (QUE) conjecture of Rudnick and Sarnak [RS] states that $\mu_{L}$ is the only semiclassical measure. This conjecture was proved for Hecke forms on arithmetic surfaces (such as the modular surface) by Lindenstrauss [L] and Soundararajan [So]. For the related setting of Eisenstein series see Luo-Sarnak [LS]and Jakobson [Ja]. For the history of the QUE conjecture we refer the reader to the reviews of Marklof [Mk], Zelditch [Ze2], and Sarnak [Sa].

In the more general setting of manifolds with Anosov geodesic flows, restrictions on possible semiclassical measures have been obtained by Anantharaman and Anantharaman-Nonnenmacher [A], [AN]; see also the papers by Rivière [Ri1], [Ri2] and Anantharaman-Silberman [AS]. In particular, [AN, Theorem 1.2] shows that every semiclassical measure on a hyperbolic surface has Kolmogorov-Sinai entropy $\geqslant \frac{1}{2}$. For comparison, the Liouville measure has entropy 1 and the delta measure on a closed geodesic has entropy 0. Examples of manifolds with ergodic but non-Anosov geodesic flows with quasimodes and eigenfunctions which violate QUE have been constructed by Donnelly [D] and Hassell $[\mathrm{H}]$; see also Faure-Nonnenmacher-de Bièvre [FNB].

Theorem 1 is in some sense orthogonal to the entropy bounds discussed above. For instance, Theorem 1 excludes the case of $\mu$ supported on a set of dimension $3-\varepsilon$, which might have entropy very close to 1 . On the other hand, it does not exclude the case

$$
\mu=\alpha \mu_{L}+(1-\alpha) \mu_{0}
$$

where $\mu_{0}$ is a delta measure on a closed geodesic and $0<\alpha \leqslant 1$, while the entropy bound excludes such measures with $\alpha<\frac{1}{2}$. Theorem 1 also does not exclude the case when $\mu$ is a countable linear combination of the measures $\delta_{\gamma_{k}}$, where $\left\{\gamma_{k}\right\}_{k=1}^{\infty}$ are all the closed geodesics: for instance,

$$
\mu=\sum_{k=1}^{\infty} 2^{-k} \delta_{\gamma_{k}}
$$

satisfies $\operatorname{supp} \mu=S^{*} M$.

Our second result is a more quantitative version of Theorem 1. 
Theorem 2. Assume that $a \in C_{0}^{\infty}\left(T^{*} M\right)$ and $\left.a\right|_{S^{*} M} \not \equiv 0$. Then, there exist constants $\mathbf{C}(a), \mathbf{h}_{0}(a)>0$ depending only on $M$ and a such that, for $0<h<\mathbf{h}_{0}(a)$ and all $u \in H^{2}(M)$,

$$
\|u\|_{L^{2}} \leqslant \mathbf{C}(a)\left\|\mathrm{Op}_{h}(a) u\right\|_{L^{2}}+\mathbf{C}(a) \frac{\log (1 / h)}{h}\left\|\left(-h^{2} \Delta-I\right) u\right\|_{L^{2}} .
$$

Theorem 1 follows immediately from Theorem 2. Indeed, take $a \in C_{0}^{\infty}\left(T^{*} M\right)$ such that $\left.a\right|_{S^{*} M} \not \equiv 0$, but $\operatorname{supp} a \cap S^{*} M \subset \mathcal{U}$. Let $u_{j}$ and $h_{j}$ satisfy (1.1). Then (1.2) implies that $\left\|\mathrm{Op}_{h_{j}}(a) u_{j}\right\|_{L^{2}} \geqslant \mathbf{C}(a)^{-1}$ for large $j$. However, if $u_{j}$ converge semiclassically to some measure $\mu$, then

$$
\left\|\mathrm{Op}_{h_{j}}(a) u_{j}\right\|_{L^{2}}^{2} \rightarrow \int_{T^{*} M}|a|^{2} d \mu \quad \text { as } j \rightarrow \infty .
$$

It follows that $\int|a|^{2} d \mu>0$, and thus $\mu(\mathcal{U})>0$.

The above argument shows that Theorem 1 still holds if we replace the requirement $\left(-h_{j}^{2} \Delta-I\right) u_{j}=0$ in $(1.1)$ by $\left\|\left(-h_{j}^{2} \Delta-I\right) u_{j}\right\|_{L^{2}}=o\left(h_{j} / \log \left(1 / h_{j}\right)\right)$, that is it applies to $o(h / \log (1 / h))$ quasimodes. This quasimode strength is almost sharp; indeed, Brooks [B], Eswarathasan-Nonnenmacher [EN], and Eswarathasan-Silberman [ES] construct a family of $\mathcal{O}(h / \log (1 / h))$ quasimodes which do not converge to $\mu_{L}$. In particular, [EN, Proposition 1.9] gives $\mathcal{O}(h / \log (1 / h))$ quasimodes which converge semiclassically to the delta measure on any given closed geodesic. We remark that the factor $(1 / h) \log (1 / h)$ in (1.2) is reminiscent of the scattering resolvent bounds on the real line for mild hyperbolic trapping; see $[\mathrm{Zw} 2, \S 3.2]$ and the references there.

Theorem 2 has applications to control for the Schrödinger equation [Ji1] and its proof can be adapted to show exponential energy decay for the damped wave equation [Ji2].

We would also like to mention a recent result of Logunov-Malinnikova [LM] giving a bound of the following form for an eigenfunction $u,\left(-h^{2} \Delta-I\right) u=0$ :

$$
\sup _{\Omega}|u| \geqslant \frac{1}{C}\left(\frac{\operatorname{vol}_{g}(\Omega)}{C}\right)^{-C / h} \sup _{M}|u|,
$$

where $C$ is a constant depending only on $M$. The bound (1.3) holds on any closed Riemannian manifold and for any subset $\Omega \subset M$ of positive volume. For hyperbolic surfaces and $\Omega$ having non-empty interior, Theorem 2 together with the unique continuation principle give the bound

$$
\|u\|_{L^{2}(\Omega)} \geqslant c_{\Omega}\|u\|_{L^{2}(M)},
$$

where $c_{\Omega}>0$ is a constant depending on $M$ and $\Omega$, but not on $h$. Unlike (1.3), the bound (1.4) cannot hold for general Riemannian manifolds: if $M$ is the round sphere and $\Omega$ lies strictly inside one hemisphere, then there exists a sequence of Gaussian beam eigenfunctions $u$ concentrating on the equator with $\|u\|_{L^{2}(\Omega)} \leqslant e^{-C / h}\|u\|_{L^{2}(M)}$. 


\subsection{Outline of the proof}

We give a rough outline of the proof of Theorem 2, assuming for simplicity that

$$
\left(-h^{2} \Delta-I\right) u=0
$$

We write

$$
u=A_{\mathcal{X}} u+A_{\mathcal{Y}} u
$$

where $A_{\mathcal{X}}$ and $A_{\mathcal{Y}}$ are constructed from two fixed pseudodifferential operators $A_{1}$ and $A_{2}$ conjugated by the wave propagator for times up to $2 \varrho \log (1 / h)$; see $(3.7)$ and $(3.16)$. The parameter $\varrho$ is chosen less than 1 but is very close to 1 ; see the remark following Proposition 3.5. The operators $A_{\mathcal{X}}$ and $A_{\mathcal{Y}}$ formally correspond to symbols $a_{\mathcal{X}}$ and $a_{\mathcal{Y}}$ such that, for some small parameter $\alpha>0$,

- for $(x, \xi) \in \operatorname{supp} a_{\mathcal{X}}$, at most $2 \alpha \log (1 / h)$ of the points

$$
\varphi_{j}(x, \xi), \quad j=0,1, \ldots, 2 \varrho \log (1 / h)
$$

lie in the set $\{a \neq 0\}$. That is, the geodesic $\varphi_{t}(x, \xi), 0 \leqslant t \leqslant 2 \varrho \log (1 / h)$ spends very little time in $\{a \neq 0\}$;

- for $(x, \xi) \in \operatorname{supp} a_{\mathcal{Y}}$, at least $\frac{1}{10} \alpha \log (1 / h)$ points (1.5) lie in the set $\{a \neq 0\}$.

To explain the intuition behind the argument, we first consider the case when $\alpha=0$, that is, for $(x, \xi) \in \operatorname{supp} a_{\mathcal{X}}$ none of the points (1.5) lie in $\{a \neq 0\}$. (In the argument for general $\alpha$ leading to (1.8), putting $\alpha=0$ is equivalent to taking $\alpha \sim 1 / \log (1 / h)$.) One can view $\{a \neq 0\}$ as a 'hole' in $S^{*} M$ and $\operatorname{supp} a_{\mathcal{X}}$ is contained in the set of 'forward trapped' geodesics (that is, those that do not go through the hole). On the other hand, points $(x, \xi)$ in supp $a_{\mathcal{Y}}$ are controlled in the sense that $\varphi_{j}(x, \xi)$ lies in the hole for some $j \in[0,2 \varrho \log (1 / h)]$. Therefore, one hopes to control $A_{\mathcal{Y}} u$ in terms of $\mathrm{Op}_{h}(a) u$ using Egorov's theorem and the fact that $u$ is an eigenfunction of the Laplacian - see (1.7) below.

The operator $A_{\mathcal{X}}$ is not pseudodifferential because it corresponds to propagation for time $2 \varrho \log (1 / h)$ which is much larger than the Ehrenfest time $\log (1 / h)$. However, conjugating $A_{\mathcal{X}}$ by the wave group we obtain a product of the form $\mathcal{A}_{-} \mathcal{A}_{+}$, where the symbols $a_{ \pm}$corresponding to $\mathcal{A}_{ \pm}$satisfy

$$
\varphi_{\mp j}\left(\operatorname{supp} a_{ \pm}\right) \cap\{a \neq 0\}=\varnothing \quad \text { for all } j=0,1, \ldots, \varrho \log (1 / h) .
$$

That is, supp $a_{-}$is 'forward trapped' and supp $a_{+}$is 'backward trapped'. The operators $\mathcal{A}_{ \pm}$lie in the calculi associated with the weak unstable/stable Lagrangian foliations on $T^{*} M \backslash\{0\}$ similar to the ones developed by Dyatlov-Zahl [DZa]; see $\S 2.3$ and the 
appendix. More precisely, the symbol $a_{+}$is regular along the weak unstable foliation and $a_{-}$is regular along the weak stable foliation. The constant curvature condition plays an important role in defining these calculi associated with Lagrangian foliations. On a general surface with negative curvature, the weak unstable/stable Lagrangian foliations are only Hölder continuous instead of smooth.

Using unique ergodicity of horocyclic flows due to Furstenberg [F], we show that $\operatorname{supp} a_{+}$is porous in the stable direction and $\operatorname{supp} a_{-}$is porous in the unstable direction (see Definition 5.6 and Lemma 5.10). Then, the fractal uncertainty principle of Bourgain-Dyatlov [DZa] implies that $\left\|\mathcal{A}_{-} \mathcal{A}_{+}\right\|_{L^{2} \rightarrow L^{2}} \leqslant C h^{\beta}$ for some $\beta>0$ and thus (see Proposition 3.5)

$$
\left\|A_{\mathcal{X}} u\right\|_{L^{2}} \leqslant C h^{\beta}\|u\|_{L^{2}}
$$

We stress that just like the operator $A_{\mathcal{X}}$, the product $\mathcal{A}_{-} \mathcal{A}_{+}$is not a pseudodifferential operator, since it corresponds to propagation for time $\varrho \log (1 / h)>\frac{1}{2} \log (1 / h)$ in both time directions. In fact, if $\mathcal{A}_{-} \mathcal{A}_{+}$were pseudodifferential with symbol $a_{-} a_{+}$, we would expect the left-hand side of (1.6) to be asymptotic to $\sup \left|a_{-} a_{+}\right|=1$. However, since $\varrho<1$ each of the operators $\mathcal{A}_{ \pm}$, corresponding to propagation for time $\varrho \log (1 / h)$ in one time direction, is still pseudodifferential in an anisotropic class; see $\S 2.3$ (but the product $\mathcal{A}_{-} \mathcal{A}_{+}$is not pseudodifferential, since the calculi in which $\mathcal{A}_{-}$and $\mathcal{A}_{+}$lie are incompatible with each other). The norm estimate (1.6) uses fractal uncertainty principle, which is a tool from harmonic analysis, and in some sense goes beyond the classical/quantum correspondence.

To estimate $A_{\mathcal{Y}} u$ in the case $\alpha=0$, we can break it into pieces, each of which corresponds to the condition $\varphi_{j}(x, \xi) \in\{a \neq 0\}$ for some $j=0,1, \ldots, 2 \varrho \log (1 / h)$. Since

$$
\left(-h^{2} \Delta-I\right) u=0
$$

$u$ is equivariant under the wave propagator; therefore, each piece can be controlled by $\mathrm{Op}_{h}(a) u$. Summing over $j$, we get

$$
\left\|A_{\mathcal{Y}} u\right\|_{L^{2}} \leqslant C \log (1 / h)\left\|\mathrm{Op}_{h}(a) u\right\|_{L^{2}}+\mathcal{O}\left(h^{\infty}\right)\|u\|_{L^{2}} .
$$

Combining (1.6) and (1.7) we get (1.2), however the term $\left\|\mathrm{Op}_{h}(a) u\right\|_{L^{2}}$ comes with an extra factor of $\log (1 / h)$. To remove this factor, we take $\alpha$ small, but positive. The estimate (1.6) still holds as long as $\alpha$ is chosen small enough depending on the fractal uncertainty exponent $\beta$, see (3.19). Moreover, we get the following improved version of (1.7) for some $\varepsilon>0$ (see Proposition 3.4; one can take $\varepsilon=\frac{1}{8}$ ):

$$
\|A \mathcal{Y} u\|_{L^{2}} \leqslant \frac{C}{\alpha}\left\|\mathrm{Op}_{h}(a) u\right\|_{L^{2}}+\mathcal{O}\left(h^{\varepsilon}\right)\|u\|_{L^{2}} .
$$

Combining (1.6) and (1.8) gives the required bound (1.2). 
The estimate (1.8) is delicate because $A_{\mathcal{Y}}$ is not pseudodifferential. To prove it, we adapt some of the methods of [A]. More precisely, if we replace $2 \varrho \log (1 / h)$ by $\tilde{\varepsilon} \log (1 / h)$ for small enough $\tilde{\varepsilon}>0$ in the definition of $A \mathcal{Y}$, then $A_{\mathcal{Y}}$ is pseudodifferential in a mildly exotic calculus, and one can use a semiclassical version of the Chebyshev inequality (see Lemma 4.6) to establish (1.8). To pass from short logarithmic times to time $2 \varrho \log (1 / h)$, we use a submultiplicative estimate; see the end of $\S 4.3$.

\section{Preliminaries}

\subsection{Dynamics of geodesic and horocyclic flows}

Let $(M, g)$ be a compact hyperbolic surface and $T^{*} M \backslash\{0\}$ consist of elements of the cotangent bundle $(x, \xi) \in T^{*} M$ such that $\xi \neq 0$. Denote by $S^{*} M=\left\{|\xi|_{g}=1\right\}$ the cosphere bundle. Define the symbol $p \in C^{\infty}\left(T^{*} M \backslash\{0\} ; \mathbb{R}\right)$ by

$$
p(x, \xi)=|\xi|_{g} .
$$

The Hamiltonian flow of $p$,

$$
\varphi_{t}:=e^{t H_{p}}: T^{*} M \backslash\{0\} \longrightarrow T^{*} M \backslash\{0\}
$$

is the homogeneous geodesic flow.

Henceforth, we assume that $M$ is orientable; if not, we may pass to a double cover of $M$. We use an explicit frame on $T^{*} M \backslash\{0\}$ consisting of four vector fields

$$
H_{p}, U_{+}, U_{-}, D \in C^{\infty}\left(T^{*} M \backslash\{0\} ; T\left(T^{*} M \backslash\{0\}\right)\right) .
$$

Here $H_{p}$ is the generator of $\varphi_{t}$ and $D=\xi \cdot \partial_{\xi}$ is the generator of dilations. The vector fields $U_{ \pm}$are defined on $S^{*} M$ as stable $\left(U_{+}\right)$and unstable $\left(U_{-}\right)$horocyclic vector fields and extended homogeneously to $T^{*} M \backslash\{0\}$, so that

$$
\left[U_{ \pm}, D\right]=\left[H_{p}, D\right]=0
$$

See for instance [DFG, formula (2.1)]. The vector fields $U_{ \pm}$are tangent to the level sets of $p$ and satisfy the commutation relations

$$
\left[H_{p}, U_{ \pm}\right]= \pm U_{ \pm}
$$

Thus, on each level set of $p$, the flow $\varphi_{t}$ has a flow/stable/unstable decomposition, with $U_{+}$spanning the stable space and $U_{-}$spanning the unstable space; see, for instance, [DFG, formula (3.14)]. We use the following notation for the weak stable/unstable spaces:

$$
L_{s}:=\operatorname{span}\left(H_{p}, U_{+}\right), L_{u}:=\operatorname{span}\left(H_{p}, U_{-}\right) \subset T\left(T^{*} M \backslash\{0\}\right) .
$$


Then, $L_{s}$ and $L_{u}$ are Lagrangian foliations; see [DZa, Lemma 4.1].

The next statement, used in $\S 5.3$ to establish the porosity condition, is a consequence of the unique ergodicity of horocyclic flows; see $[\mathrm{F}],[\mathrm{Mc}],[\mathrm{Ra}],[\mathrm{C}],[\mathrm{HM}]$.

Proposition 2.1. Let $\mathcal{U} \subset S^{*} M$ be a non-empty open set. Then, there exists $T>0$ depending only on $M$ and $\mathcal{U}$ such that, for all $(x, \xi) \in S^{*} M$,

$$
\left\{e^{s U_{ \pm}}(x, \xi): 0 \leqslant s \leqslant T\right\} \cap \mathcal{U} \neq \varnothing .
$$

Proof. We focus on the case of $U_{+}$; the same proof applies to $U_{-}$. Denote by $\mu_{L}$ the Liouville probability measure on $S^{*} M$. By the unique ergodicity of the horocyclic flow $e^{s U_{+}}, \mu_{L}$ is the only probability measure on $S^{*} M$ invariant under $e^{s U_{+}}$.

Let $f \in C\left(S^{*} M\right)$ be a continuous function. Then, we have uniform convergence

$$
\langle f\rangle_{T}:=\frac{1}{T} \int_{0}^{T} f \circ e^{s U_{+}} d s \rightarrow\langle f\rangle_{\mu}:=\int_{S^{*} M} f d \mu_{L} \quad \text { as } T \rightarrow \infty .
$$

Indeed, assume that (2.8) is false. Then, there exist $\varepsilon>0$ and sequences $T_{k} \rightarrow \infty,\left(x_{k}, \xi_{k}\right) \in$ $S^{*} M$ such that

$$
\left|\langle f\rangle_{T_{k}}\left(x_{k}, \xi_{k}\right)-\langle f\rangle_{\mu}\right| \geqslant \varepsilon
$$

Consider the probability measures $\nu_{k}$ on $S^{*} M$ defined by

$$
\int_{S^{*} M} g d \nu_{k}=\langle g\rangle_{T_{k}}\left(x_{k}, \xi_{k}\right) \quad \text { for all } g \in C\left(S^{*} M\right) .
$$

Passing to a subsequence, we may assume that $\nu_{k}$ converge weakly to some probability measure $\nu$. Since $T_{k} \rightarrow \infty$, the measure $\nu$ is invariant under the flow $e^{s U_{+}}$, thus $\nu=\mu_{L}$. However, $\int f d \nu \neq \int f d \mu_{L}$ by (2.9), giving a contradiction. This finishes the proof of (2.8).

Now, choose $f \in C\left(S^{*} M\right)$ such that

$$
\operatorname{supp} f \subset \mathcal{U} \quad \text { and }\langle f\rangle_{\mu}=1 \text {. }
$$

By (2.8), there exists $T>0$ such that $\langle f\rangle_{T}>\frac{1}{2}$ everywhere. This implies (2.7).

\subsection{Operators and propagation}

We use the standard classes of semiclassical pseudodifferential operators with classical symbols $\Psi_{h}^{k}(M)$, with $\Psi_{h}^{\text {comp }}(M)$ denoting operators $A \in \Psi_{h}^{k}(M)$ such that the wavefront set $\mathrm{WF}_{h}(A)$ is a compact subset of $T^{*} M$. We refer the reader to the book of Zworski [Zw1] for an introduction to semiclassical analysis used in this paper, to [Zw1, §14.2.2] for 
pseudodifferential operators on manifolds, and to [DZw, §E.1.5] and [DZa, §2.1] for the classes $\Psi_{h}^{k}(M)$ used here. Denote by $S^{k}\left(T^{*} M\right)$ the corresponding symbol classes, and by

$$
\sigma_{h}: \Psi_{h}^{k}(M) \rightarrow S^{k}\left(T^{*} M\right) \quad \text { and } \quad \mathrm{Op}_{h}: S^{k}\left(T^{*} M\right) \rightarrow \Psi_{h}^{k}(M)
$$

the principal symbol map and a (non-canonical) quantization map. For $A, B \in \Psi_{h}^{k}(M)$ and an open set $U \subset T^{*} M$, we say that $A=B+\mathcal{O}\left(h^{\infty}\right)$ microlocally on $U$, if

$$
\mathrm{WF}_{h}(A-B) \cap U=\varnothing \text {. }
$$

We have the following norm bound:

$$
A \in \Psi_{h}^{0}(M), \quad \sup \left|\sigma_{h}(A)\right| \leqslant 1 \quad \Longrightarrow \quad\|A\|_{L^{2} \rightarrow L^{2}} \leqslant 1+C h .
$$

Indeed, applying the sharp Gårding inequality [Zw1, Theorem 4.32] to the operator $I-A^{*} A$, we get, for all $u \in L^{2}(M)$,

$$
\|u\|_{L^{2}}^{2}-\|A u\|_{L^{2}}^{2}=\left\langle\left(I-A^{*} A\right) u, u\right\rangle_{L^{2}} \geqslant-C h\|u\|_{L^{2}}^{2}
$$

which gives (2.10).

The operator $-h^{2} \Delta$ lies in $\Psi_{h}^{2}(M)$ and, with $p$ defined in (2.1),

$$
\sigma_{h}\left(-h^{2} \Delta\right)=p^{2}
$$

For us it will be convenient to have an operator with principal symbol $p$, since the corresponding Hamiltonian flow is homogeneous. Of course, we have to cut away from the zero section, as $p$ is not smooth there. We thus fix a function

$$
\psi_{P} \in C_{0}^{\infty}((0, \infty) ; \mathbb{R}), \quad \psi_{P}(\lambda)=\sqrt{\lambda} \quad \text { for } \frac{1}{16} \leqslant \lambda \leqslant 16,
$$

and define the operator

$$
P:=\psi_{P}\left(-h^{2} \Delta\right), \quad P^{*}=P .
$$

By the functional calculus of pseudodifferential operators (see [Zw1, Theorem 14.9] or [DS, §8]), we have

$$
P \in \Psi_{h}^{\mathrm{comp}}(M), \quad \sigma_{h}(P)=p \text { on }\left\{\frac{1}{4} \leqslant|\xi|_{g} \leqslant 4\right\} .
$$

To quantize the flow $\varphi_{t}$, we use the propagator

$$
U(t):=e^{-i t P / h}: L^{2}(M) \longrightarrow L^{2}(M)
$$

The operator $U(t)$ is unitary on $L^{2}(M)$.

For a bounded operator $A: L^{2}(M) \rightarrow L^{2}(M)$, define

$$
A(t):=U(-t) A U(t) .
$$

If $A \in \Psi_{h}^{\text {comp }}(M), \mathrm{WF}_{h}(A) \subset\left\{\frac{1}{4}<|\xi|_{g}<4\right\}$, and $t$ is bounded uniformly in $h$, then Egorov's theorem [Zw1, Theorem 11.1] implies that

$$
A(t) \in \Psi_{h}^{\mathrm{comp}}(M) \quad \text { and } \quad \sigma_{h}(A(t))=\sigma_{h}(A) \circ \varphi_{t} .
$$




\subsection{Anisotropic calculi and long-time propagation}

If $A \in \Psi_{h}^{\text {comp }}(M)$ and $t$ grows with $h$, then $A(t)$ will generally not be pseudodifferential in the class $\Psi_{h}^{\text {comp }}$, since the derivatives of the symbol $\sigma_{h}(A) \circ \varphi_{t}$ may grow exponentially with $t$. In this section, we introduce a more general calculus which contains the operators $A(t)$ for $|t| \leqslant \varrho \log (1 / h), \varrho<1$. (More precisely, we will have two calculi, one of which works for $t \geqslant 0$, and the other for $t \leqslant 0$.) Our calculus is similar to the one developed in [DZa, $\S 3]$, with remarks on the differences of these two calculi and the proofs of some of the properties of the calculus contained the appendix.

Fix $\varrho \in[0,1)$ and let $L \in\left\{L_{u}, L_{s}\right\}$, where the Lagrangian foliations $L_{u}$ and $L_{s}$ are defined in (2.6). Define the class of $h$-dependent symbols $S_{L, \varrho}^{\text {comp }}\left(T^{*} M \backslash\{0\}\right)$ as follows: $a \in S_{L, \varrho}^{\mathrm{comp}}\left(T^{*} M \backslash\{0\}\right)$ if

(1) $a(x, \xi ; h)$ is smooth in $(x, \xi) \in T^{*} M \backslash\{0\}$, defined for $0<h \leqslant 1$, and supported in an $h$-independent compact subset of $T^{*} M \backslash\{0\}$;

(2) $\sup _{x, \xi}|a(x, \xi ; h)| \leqslant C$ for some constant $C$ and all $h$;

(3) a satisfies the derivative bounds

$$
\sup _{x, \xi}\left|Y_{1} \ldots Y_{m} Z_{1} \ldots Z_{k} a(x, \xi ; h)\right| \leqslant C h^{-\varrho k-\varepsilon}, \quad 0<h \leqslant 1,
$$

for all $\varepsilon>0$ and all vector fields $Y_{1}, \ldots, Y_{m}, Z_{1}, \ldots, Z_{k}$ on $T^{*} M \backslash\{0\}$ such that $Y_{1}, \ldots, Y_{m}$ are tangent to $L$. Here the constant $C$ depends on $Y_{1}, \ldots, Y_{m}, Z_{1}, \ldots, Z_{k}$ and $\varepsilon$, but does not depend on $h$.

This class is slightly larger than the one in [DZa, Definition 3.2], because we require (2.16) to hold for all $\varepsilon>0$, while [DZa] had $\varepsilon:=0$.

We use the following notation:

$$
f(h)=\mathcal{O}\left(h^{\alpha-}\right) \quad \text { if } f(h)=\mathcal{O}\left(h^{\alpha-\varepsilon}\right) \text { for all } \varepsilon>0 .
$$

In terms of the frame (2.3), the derivative bounds (2.16) become

$$
\begin{array}{ll}
\sup _{x, \xi}\left|H_{p}^{k} U_{+}^{\ell} U_{-}^{m} D^{n} a(x, \xi ; h)\right|=\mathcal{O}\left(h^{-\varrho(m+n)-}\right) & \text { for } L=L_{s}, \\
\sup _{x, \xi}\left|H_{p}^{k} U_{-}^{\ell} U_{+}^{m} D^{n} a(x, \xi ; h)\right|=\mathcal{O}\left(h^{-\varrho(m+n)-}\right) & \text { for } L=L_{u} .
\end{array}
$$

If $a \in C_{0}^{\infty}\left(T^{*} M \backslash\{0\}\right)$ is an $h$-independent symbol, then it follows from the commutation relations (2.4) and (2.5) that

$$
H_{p}^{k} U_{+}^{\ell} U_{-}^{m} D^{n}\left(a \circ \varphi_{t}\right)=e^{(m-\ell) t}\left(H_{p}^{k} U_{+}^{\ell} U_{-}^{m} D^{n} a\right) \circ \varphi_{t} .
$$

Therefore,

$$
a \circ \varphi_{t} \in S_{L_{s}, \varrho}^{\mathrm{comp}}\left(T^{*} M \backslash\{0\}\right) \text { uniformly in } t, \quad 0 \leqslant t \leqslant \varrho \log (1 / h) .
$$


Similarly,

$$
a \circ \varphi_{-t} \in S_{L_{u}, \varrho}^{\mathrm{comp}}\left(T^{*} M \backslash\{0\}\right) \text { uniformly in } t, \quad 0 \leqslant t \leqslant \varrho \log (1 / h) .
$$

Let $\Psi_{h, L, \varrho}^{\text {comp }}\left(T^{*} M \backslash\{0\}\right), L \in\left\{L_{u}, L_{s}\right\}$, be the classes of pseudodifferential operators with symbols in $S_{L, \varrho}^{\text {comp }}$ defined following the same construction as in [DZa, $\left.§ 3\right]$. They satisfy similar properties to the operators used in $[\mathrm{DZa}]$, in particular they are pseudolocal and bounded on $L^{2}(M)$ uniformly in $h$. However, the $\mathcal{O}\left(h^{1-\varrho}\right)$ remainders have to be replaced by $\mathcal{O}\left(h^{1-\varrho-}\right)$ because of the relaxed assumptions on derivatives (2.16). We denote by

$$
\begin{aligned}
\mathrm{Op}_{h}^{L}: S_{L, \varrho}^{\mathrm{comp}}\left(T^{*} M \backslash\{0\}\right) & \longrightarrow \Psi_{h, L, \varrho}^{\mathrm{comp}}\left(T^{*} M \backslash\{0\}\right), \\
a & \longmapsto \mathrm{Op}_{h}^{L}(a),
\end{aligned}
$$

a (non-canonical) quantization procedure. See $\S$ A.4 for more details.

The $\Psi_{h, L, \varrho}^{\text {comp }}$ calculus satisfies a version of Egorov's theorem (Lemma A.8). It states that, for $A=\mathrm{Op}_{h}(a)$, where $a \in C_{0}^{\infty}\left(\left\{\frac{1}{4}<|\xi|_{g}<4\right\}\right)$ is independent of $h$,

$$
\begin{gathered}
A(t)=\mathrm{Op}_{h}^{L_{s}}\left(a \circ \varphi_{t}\right)+\mathcal{O}\left(h^{1-\varrho-}\right)_{L^{2} \rightarrow L^{2}}, \\
A(-t)=\mathrm{Op}_{h}^{L_{u}}\left(a \circ \varphi_{-t}\right)+\mathcal{O}\left(h^{1-\varrho-}\right)_{L^{2} \rightarrow L^{2}}
\end{gathered}
$$

uniformly in $t \in[0, \varrho \log (1 / h)]$.

\section{Proof of Theorem 2}

In this section we give the proof of Theorem 2. It uses two key estimates, Proposition 3.4 and Proposition 3.5, which are proved in $\S 4$ and $\S 5$ respectively.

\subsection{Partitions and words}

We assume that $a \in C_{0}^{\infty}\left(T^{*} M\right)$ and $\left.a\right|_{S^{*} M} \not \equiv 0$ as in the assumptions of Theorem 2. Fix conic open sets

$$
\mathcal{U}_{1}, \mathcal{U}_{2} \subset T^{*} M \backslash\{0\}, \quad \mathcal{U}_{1}, \mathcal{U}_{2} \neq \varnothing, \quad \overline{\mathcal{U}}_{1} \cap \overline{\mathcal{U}}_{2}=\varnothing, \quad \overline{\mathcal{U}}_{2} \cap S^{*} M \subset\{a \neq 0\} .
$$

(The sets $\mathcal{U}_{j}$ and conditions (3.2) below are used in the proof of Proposition 3.5.)

We introduce a pseudodifferential partition of unity

$$
I=A_{0}+A_{1}+A_{2}, \quad A_{0} \in \Psi_{h}^{0}(M) \text { and } A_{1}, A_{2} \in \Psi_{h}^{\mathrm{comp}}(M)
$$


such that the following conditions are satisfied (see Figure 1).

- $A_{0}$ is microlocalized away from the cosphere bundle $S^{*} M$. More specifically, we put $A_{0}:=\psi_{0}\left(-h^{2} \Delta\right)$, where $\psi_{0} \in C^{\infty}(\mathbb{R} ;[0,1])$ satisfies

$$
\operatorname{supp} \psi_{0} \cap\left[\frac{1}{4}, 4\right]=\varnothing \quad \text { and } \quad \operatorname{supp}\left(1-\psi_{0}\right) \subset\left(\frac{1}{16}, 16\right) \text {. }
$$

This implies that

$$
\mathrm{WF}_{h}\left(A_{0}\right) \cap\left\{\frac{1}{2} \leqslant|\xi|_{g} \leqslant 2\right\}=\varnothing \quad \text { and } \quad \mathrm{WF}_{h}\left(I-A_{0}\right) \subset\left\{\frac{1}{4}<|\xi|_{g}<4\right\}
$$

- $A_{1}$ and $A_{2}$ are microlocalized in an energy shell and away from $\mathcal{U}_{1}$ and $\mathcal{U}_{2}$, that is

$$
\mathrm{WF}_{h}\left(A_{1}\right) \cup \mathrm{WF}_{h}\left(A_{2}\right) \subset\left\{\frac{1}{4}<|\xi|_{g}<4\right\}
$$

and

$$
\mathrm{WF}_{h}\left(A_{1}\right) \cap \overline{\mathcal{U}}_{1}=\mathrm{WF}_{h}\left(A_{2}\right) \cap \overline{\mathcal{U}}_{2}=\varnothing
$$

- $A_{1}$ is controlled by $a$ on the cosphere bundle, that is

$$
\mathrm{WF}_{h}\left(A_{1}\right) \cap S^{*} M \subset\{a \neq 0\} .
$$

To construct $A_{1}$ and $A_{2}$, note that (3.1)-(3.3) are equivalent to $\mathrm{WF}_{h}\left(A_{j}\right) \subset \Omega_{j}$, where

$$
\Omega_{1}:=\left(\left\{\frac{1}{4}<|\xi|_{g}<4\right\} \backslash \overline{\mathcal{U}}_{1}\right) \cap\left(\{a \neq 0\} \cup\left(T^{*} M \backslash S^{*} M\right)\right)
$$

and

$$
\Omega_{2}:=\left\{\frac{1}{4}<|\xi|_{g}<4\right\} \backslash \overline{\mathcal{U}}_{2}
$$

are open subsets of $T^{*} M$ such that

$$
\mathrm{WF}_{h}\left(I-A_{0}\right) \subset\left\{\frac{1}{4}<|\xi|_{g}<4\right\} \subset \Omega_{1} \cup \Omega_{2}
$$

It remains to use a pseudodifferential partition of unity to find $A_{1}$ and $A_{2}$ such that (3.1)(3.3) hold and $A_{1}+A_{2}=I-A_{0}$. (For instance, one can write $I-A_{0}=\mathrm{Op}_{h}(b)+\mathcal{O}\left(h^{\infty}\right)$, where supp $b \subset \Omega_{1} \cup \Omega_{2}$, split $b=a_{1}+a_{2}$ for some symbols $a_{1}$ and $a_{2}$ with supp $a_{j} \subset \Omega_{j}$, and put $A_{j}:=\mathrm{Op}_{h}\left(a_{j}\right)$.) We moreover choose $A_{1}$ and $A_{2}$ so that

$$
0 \leqslant a_{\ell} \leqslant 1 \quad \text { where } a_{\ell}:=\sigma_{h}\left(A_{\ell}\right), \ell=0,1,2 .
$$

We next dynamically refine the partition $A_{j}$. For each $n \in \mathbb{N}_{0}$, define the set of words of length $n$,

$$
\mathcal{W}(n):=\{1,2\}^{n}=\left\{\mathbf{w}=w_{0} \ldots w_{n-1}: w_{0}, \ldots, w_{n-1} \in\{1,2\}\right\}
$$




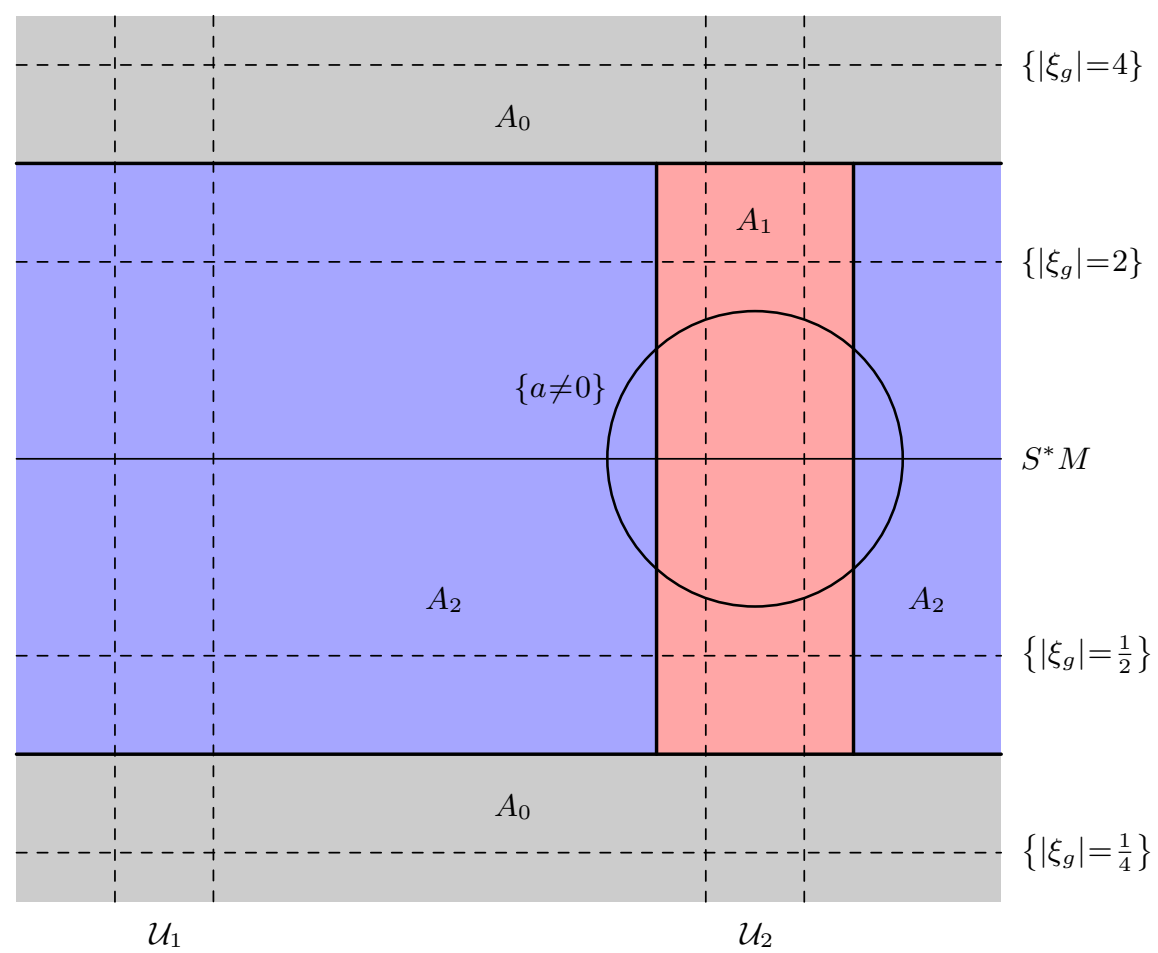

Figure 1. The sets $\mathcal{U}_{1}, \mathcal{U}_{2}, \mathrm{WF}_{h}\left(A_{j}\right)$ (shaded), and $\{a \neq 0\}$ inside $T^{*} M$. The vertical direction corresponds to dilating $\xi$.

For each word $\mathbf{w}=w_{0} \ldots w_{n-1} \in \mathcal{W}(n)$, using the notation (2.14), define the operator

$$
A_{\mathbf{w}}=A_{w_{n-1}}(n-1) A_{w_{n-2}}(n-2) \ldots A_{w_{1}}(1) A_{w_{0}}(0) .
$$

If $n$ is bounded independently of $h$, then, as a consequence of Egorov's theorem (2.15), we have $A_{\mathbf{w}} \in \Psi_{h}^{\text {comp }}(M)$ and $\sigma_{h}\left(A_{\mathbf{w}}\right)=a_{\mathbf{w}}$, where

$$
a_{\mathbf{w}}=\prod_{j=0}^{n-1}\left(a_{w_{j}} \circ \varphi_{j}\right) .
$$

For a subset $\mathcal{E} \subset \mathcal{W}(n)$, define the operator $A_{\mathcal{E}}$ and the symbol $a_{\mathcal{E}}$ by

$$
A_{\mathcal{E}}:=\sum_{\mathbf{w} \in \mathcal{E}} A_{\mathbf{w}} \quad \text { and } \quad a_{\mathcal{E}}:=\sum_{\mathbf{w} \in \mathcal{E}} a_{\mathbf{w}} .
$$

Since $A_{1}+A_{2}=I-A_{0}$ and $P$ are both functions of $\Delta$, they commute with each other. Therefore, $A_{1}+A_{2}$ commutes with $U(t)$, which implies

$$
A_{\mathcal{W}(n)}=\left(A_{1}+A_{2}\right)^{n}
$$

This operator is equal to the identity microlocally near $S^{*} M$, implying the following lemma. 
Lemma 3.1. We have, for all $n \geqslant 0$ and $u \in H^{2}(M)$,

$$
\left\|u-\left(A_{1}+A_{2}\right)^{n} u\right\|_{L^{2}} \leqslant C\left\|\left(-h^{2} \Delta-I\right) u\right\|_{L^{2}} .
$$

Proof. Since $A_{1}+A_{2}=I-A_{0}=I-\psi_{0}\left(-h^{2} \Delta\right)$, we have

$$
u-\left(A_{1}+A_{2}\right)^{n} u=\psi_{1}\left(-h^{2} \Delta\right)\left(-h^{2} \Delta-I\right) u, \quad \text { where } \psi_{1}(\lambda):=\frac{1-\left(1-\psi_{0}(\lambda)\right)^{n}}{\lambda-1} .
$$

Since $1 \notin \operatorname{supp} \psi_{0}$, we have $\sup _{\lambda \in \mathbb{R}}\left|\psi_{1}(\lambda)\right| \leqslant C$ for some constant $C$ independent of $n$, and (3.9) follows.

\subsection{Long words and key estimates}

Take $\varrho \in(0,1)$ very close to 1 , to be chosen later (in Proposition 3.5 ), and put

$$
N_{0}:=\left\lceil\frac{1}{4} \varrho \log (1 / h)\right\rceil \in \mathbb{N} \text { and } N_{1}:=4 N_{0} \approx \varrho \log (1 / h)
$$

Then, words of length $N_{0}$ and $N_{1}$ give rise to pseudodifferential operators in the calculus $\Psi_{h, L, \varrho}^{\text {comp }}$ discussed in $\S 2.3$.

Lemma 3.2. For each $\mathbf{w} \in \mathcal{W}\left(N_{0}\right)$ we have (with bounds independent of $\mathbf{w}$ )

$$
a_{\mathbf{w}} \in S_{L_{s}, \varrho / 4}^{\mathrm{comp}}\left(T^{*} M \backslash\{0\}\right) \quad \text { and } \quad A_{\mathbf{w}}=\mathrm{Op}_{h}^{L_{s}}\left(a_{\mathbf{w}}\right)+\mathcal{O}\left(h^{3 / 4}\right)_{L^{2} \rightarrow L^{2}} .
$$

If instead $\mathbf{w} \in \mathcal{W}\left(N_{1}\right)$, then

$$
a_{\mathbf{w}} \in S_{L_{s}, \varrho}^{\mathrm{comp}}\left(T^{*} M \backslash\{0\}\right) \quad \text { and } \quad A_{\mathbf{w}}=\mathrm{Op}_{h}^{L_{s}}\left(a_{\mathbf{w}}\right)+\mathcal{O}\left(h^{1-\varrho-}\right)_{L^{2} \rightarrow L^{2}} .
$$

Proof. We prove (3.11); the proof of (3.10) is identical, replacing $\varrho$ by $\frac{1}{4} \varrho$. First of all, by (2.19) and (3.4) we have, uniformly in $j=0, \ldots, N_{1}-1$,

$$
a_{w_{j}} \circ \varphi_{j} \in S_{L_{s}, \varrho}^{\mathrm{comp}}\left(T^{*} M \backslash\{0\}\right) \quad \text { and } \quad \sup \left|a_{w_{j}} \circ \varphi_{j}\right| \leqslant 1
$$

Recalling the definition (3.6), we have $a_{\mathbf{w}} \in S_{L_{s}, \varrho}^{\mathrm{comp}}\left(T^{*} M \backslash\{0\}\right)$, by Lemma A.1, where we put $a_{j}:=a_{w_{j}} \circ \varphi_{j}$. Here, we use the relation (A.2) of the classes $S_{L_{s}, \varrho, \varrho^{\prime}}^{\text {comp }}$ used in the appendix to the class $S_{L_{s}, \varrho}^{\text {comp }}$ used here. Next, by Lemma A.8 we have, uniformly in $j=0, \ldots, N_{1}-1$,

$$
A_{w_{j}}(j)=\mathrm{Op}_{h}^{L_{s}}\left(a_{w_{j}} \circ \varphi_{j}\right)+\mathcal{O}\left(h^{1-\varrho-}\right)_{L^{2} \rightarrow L^{2}} .
$$

Applying Lemma A.6 with $A_{j}:=A_{w_{j}}(j)$, we get $A_{\mathbf{w}}=\mathrm{Op}_{h}^{L_{s}}\left(a_{\mathbf{w}}\right)+\mathcal{O}\left(h^{1-\varrho-}\right)_{L^{2} \rightarrow L^{2}}$. 
Now, define the density function $F: \mathcal{W}\left(N_{0}\right) \rightarrow[0,1]$ by

$$
F\left(w_{0} \ldots w_{N_{0}-1}\right)=\frac{\#\left\{j \in\left\{0, \ldots, N_{0}-1\right\} \mid w_{j}=1\right\}}{N_{0}} .
$$

Fix small $\alpha \in(0,1)$ to be chosen later (in $(3.21)$ ), and define

$$
\mathcal{Z}:=\{F \geqslant \alpha\} \subset \mathcal{W}\left(N_{0}\right)
$$

We call the words $\mathbf{w} \in \mathcal{Z}$ controlled because for each $(x, \xi) \in \operatorname{supp} a_{\mathbf{w}}$, at least $\alpha N_{0}$ of the points $\varphi_{0}(x, \xi), \varphi_{1}(x, \xi), \ldots, \varphi_{N_{0}-1}(x, \xi)$ lie in $\operatorname{supp} a_{1}$ and, due to $(3.3)$, are controlled by $a$.

We chose $N_{0}$ short enough so that the operators $A_{\mathbf{w}}, \mathbf{w} \in \mathcal{W}\left(N_{0}\right)$ are pseudodifferential and Egorov's theorem (3.10) holds with remainder $\mathcal{O}\left(h^{3 / 4}\right)$. This will be convenient for the estimates in $\S 4$ below, in particular in Lemma 4.4 (explaining why we did not replace $N_{0}$ by $N_{1}$ ). However, to apply the fractal uncertainty principle (Proposition 3.5), we need to propagate for time $2 N_{1}=8 N_{0} \approx 2 \varrho \log (1 / h)$. To bridge the resulting gap, we define the set of controlled words $\mathcal{Y} \subset \mathcal{W}\left(2 N_{1}\right)$ by iterating $\mathcal{Z}$. More specifically, writing words in $\mathcal{W}\left(2 N_{1}\right)$ as concatenations $\mathbf{w}^{(1)} \ldots \mathbf{w}^{(8)}$, where $\mathbf{w}^{(1)}, \ldots, \mathbf{w}^{(8)} \in \mathcal{W}\left(N_{0}\right)$, define the partition

$$
\mathcal{W}\left(2 N_{1}\right)=\mathcal{X} \sqcup \mathcal{Y}
$$

where

$$
\begin{aligned}
& \mathcal{X}:=\left\{\mathbf{w}^{(1)} \ldots \mathbf{w}^{(8)}: \mathbf{w}^{(\ell)} \notin \mathcal{Z} \text { for all } \ell\right\}, \\
& \mathcal{Y}:=\left\{\mathbf{w}^{(1)} \ldots \mathbf{w}^{(8)}: \mathbf{w}^{(\ell)} \in \mathcal{Z} \text { for some } \ell\right\} .
\end{aligned}
$$

In our argument, the parameter $\alpha$ will be taken small so that $\mathcal{X}$ has few elements. The size of $\mathcal{X}$ is estimated by the following statement (which is not sharp, but provides a bound sufficient for us).

LEMma 3.3. The number of elements in $\mathcal{X}$ is bounded by

$$
\# \mathcal{X} \leqslant C h^{-4 \sqrt{\alpha}}
$$

(here $C$ may depend on $\alpha$ ).

Proof. The complement $\mathcal{W}\left(N_{0}\right) \backslash \mathcal{Z}$ consists of words $\mathbf{w}=w_{0} \ldots w_{N_{0}-1}, w_{j} \in\{1,2\}$, such that the set $S_{\mathbf{w}}=\left\{j: w_{j}=1\right\}$ has no more than $\left\lfloor\alpha N_{0}\right\rfloor$ elements. We add arbitrary elements to the set $S_{\mathbf{w}}$ to ensure that it has size exactly $\left\lfloor\alpha N_{0}\right\rfloor$. Each choice of $S_{\mathbf{w}}$ corresponds to at most $2^{\alpha N_{0}} \leqslant h^{-\alpha / 4}$ words $\mathbf{w}$, and, by Stirling's formula,

$$
\#\left\{S_{\mathbf{w}}: \mathbf{w} \in \mathcal{W}\left(N_{0}\right) \backslash \mathcal{Z}\right\} \leqslant\left(\begin{array}{c}
N_{0} \\
\left\lfloor\alpha N_{0}\right\rfloor
\end{array}\right) \leqslant C e^{-(\alpha \log \alpha+(1-\alpha) \log (1-\alpha)) N_{0}} .
$$


Since $-(\alpha \log \alpha+(1-\alpha) \log (1-\alpha)) \leqslant \sqrt{\alpha}$ for $0 \leqslant \alpha \leqslant 1$, we have

$$
\#\left(\mathcal{W}\left(N_{0}\right) \backslash \mathcal{Z}\right) \leqslant C h^{-\alpha / 4-\sqrt{\alpha} / 4} \leqslant C h^{-\sqrt{\alpha} / 2} .
$$

As $\# \mathcal{X}=\#\left(\mathcal{W}\left(N_{0}\right) \backslash \mathcal{Z}\right)^{8}$, we obtain $(3.17)$.

Now we state the two key estimates used in the proof. The first one, proved in $\S 4$, estimates the mass of an approximate eigenfunction on the controlled region $\mathcal{Y}$.

Proposition 3.4. We have, for all $u \in H^{2}(M)$, with $A \mathcal{y}$ defined by (3.7),

$$
\left\|A_{\mathcal{Y}} u\right\|_{L^{2}} \leqslant \frac{C}{\alpha}\left\|\mathrm{Op}_{h}(a) u\right\|_{L^{2}}+\frac{C \log (1 / h)}{\alpha h}\left\|\left(-h^{2} \Delta-I\right) u\right\|_{L^{2}}+\mathcal{O}\left(h^{1 / 8}\right)\|u\|_{L^{2}}
$$

where the constant $C$ does not depend on $\alpha$.

The second estimate, proved in $\S 5$ using a fractal uncertainty principle, is a norm bound on the operator corresponding to every single word of length $2 N_{1} \approx 2 \varrho \log (1 / h)$ :

Proposition 3.5. There exist $\beta>0, \varrho \in(0,1)$ depending only on $M, \mathcal{U}_{1}$ and $\mathcal{U}_{2}$ such that

$$
\sup _{\mathbf{w} \in \mathcal{W}\left(2 N_{1}\right)}\left\|A_{\mathbf{w}}\right\|_{L^{2} \rightarrow L^{2}} \leqslant C h^{\beta}
$$

Remark. Since the proof of [BD, Proposition 4.2] uses the triangle inequality, the estimate on the norm of $A_{\mathbf{w}}$ is $\mathcal{O}\left(h^{\tilde{\beta}-2(1-\varrho)}\right)$ for some $\tilde{\beta}>0$ depending on $M, \mathcal{U}_{1}$ and $\mathcal{U}_{2}$, and thus $\varrho$ has to be close enough to 1 depending on $\tilde{\beta}$ to get decay of this norm. On the other hand, we cannot put $\varrho=1$, since the calculus described in $\S 2.3$ only works for $\varrho<1$.

\subsection{End of the proof of Theorem 2}

Take $\beta$ and $\varrho$ from Proposition 3.5. We may assume that $\beta<\frac{1}{8}$. Since

$$
A_{\mathcal{X}}+A_{\mathcal{Y}}=A_{\mathcal{W}\left(2 N_{1}\right)}=\left(A_{1}+A_{2}\right)^{2 N_{1}}
$$

by (3.8), for all $u \in H^{2}(M)$ we have

$$
\|u\|_{L^{2}} \leqslant\left\|A_{\mathcal{X}} u\right\|_{L^{2}}+\left\|A_{\mathcal{Y}} u\right\|_{L^{2}}+\left\|u-\left(A_{1}+A_{2}\right)^{2 N_{1}} u\right\|_{L^{2}} .
$$

Combining Lemma 3.3 with Proposition 3.5 and using the triangle inequality, we have

$$
\left\|A_{\mathcal{X}} u\right\|_{L^{2}}=\mathcal{O}\left(h^{\beta-4 \sqrt{\alpha}}\right)\|u\|_{L^{2}} .
$$

Combining this with Proposition 3.4 and Lemma 3.1 we obtain

$$
\|u\|_{L^{2}} \leqslant \frac{C}{\alpha}\left\|\mathrm{Op}_{h}(a) u\right\|_{L^{2}}+\frac{C \log (1 / h)}{\alpha h}\left\|\left(-h^{2} \Delta-I\right) u\right\|_{L^{2}}+\mathcal{O}\left(h^{\beta-4 \sqrt{\alpha}}\right)\|u\|_{L^{2}}
$$

Choosing

$$
\alpha:=\frac{1}{64} \beta^{2}, \quad \text { so that } \beta-4 \sqrt{\alpha}=\frac{1}{2} \beta,
$$

and taking $h$ small enough to remove the $\mathcal{O}\left(h^{\beta / 2}\right)$ term on the right-hand side of (3.20), we obtain (1.2), finishing the proof. 


\section{The controlled region}

In this section we prove Proposition 3.4, estimating an approximate eigenfunction $u$ on geodesics which spend a positive fraction of their time inside $\{a \neq 0\}$. The proof uses tools similar to $[\mathrm{A}, \S 2]$.

\subsection{Control and propagation}

Recall the operator $A_{1} \in \Psi_{h}^{\mathrm{comp}}(M)$ constructed in $\S 3.1$. We first use the wavefront set restriction (3.3) to estimate $A_{1} u$.

LEMMA 4.1. For all $u \in H^{2}(M)$ we have

$$
\left\|A_{1} u\right\|_{L^{2}} \leqslant C\left\|\mathrm{Op}_{h}(a) u\right\|_{L^{2}}+C\left\|\left(-h^{2} \Delta-I\right) u\right\|_{L^{2}}+C h\|u\|_{L^{2}} .
$$

Proof. By (3.3) we have supp $a_{1} \cap S^{*} M \subset\{a \neq 0\}$, where $a_{1}=\sigma_{h}\left(A_{1}\right)$. As $p^{2}-1$ is a defining function for $S^{*} M$, there exist $b, q \in C_{0}^{\infty}\left(T^{*} M\right)$ such that $a_{1}=a b+q\left(p^{2}-1\right)$. It follows that

$$
A_{1}=\mathrm{Op}_{h}(b) \mathrm{Op}_{h}(a)+\mathrm{Op}_{h}(q)\left(-h^{2} \Delta-I\right)+\mathcal{O}(h)_{L^{2} \rightarrow L^{2}} .
$$

It remains to apply (4.2) to $u$ and use the fact that $\mathrm{Op}_{h}(b)$ and $\mathrm{Op}_{h}(q)$ are bounded on $L^{2}$ uniformly in $h$.

Next, if we control $A u$ for some operator $A$, then we also control $A(t) u$, where $A(t)$ is defined using (2.14).

Lemma 4.2. Assume that $A: L^{2}(M) \rightarrow L^{2}(M)$ is bounded uniformly in $h$. Then, there exists a constant $C$ such that, for all $t \in \mathbb{R}$ and $u \in H^{2}(M)$,

$$
\|A(t) u\|_{L^{2}} \leqslant\|A u\|_{L^{2}}+\frac{C|t|}{h}\left\|\left(-h^{2} \Delta-I\right) u\right\|_{L^{2}}
$$

Proof. Recall from (2.14) that $A(t)=U(-t) A U(t)$, where $U(t)=e^{-i t P / h}$ and the operator $P \in \Psi_{h}^{\text {comp }}(M)$ is defined in (2.11). Since

$$
\partial_{t}\left(e^{i t / h} U(t)\right)=-\frac{i}{h} e^{i t / h} U(t)(P-I),
$$

integrating from 0 to $t$, we have

$$
\left\|U(t) u-e^{-i t / h} u\right\|_{L^{2}}=\left\|e^{i t / h} U(t) u-u\right\|_{L^{2}} \leqslant \frac{|t|}{h}\|(P-I) u\|_{L^{2}} .
$$

Then,

$$
\|A(t) u\|_{L^{2}}=\|A U(t) u\|_{L^{2}} \leqslant\|A u\|_{L^{2}}+\frac{C|t|}{h}\|(P-I) u\|_{L^{2}} .
$$


We have $P-I=\psi_{E}\left(-h^{2} \Delta\right)\left(-h^{2} \Delta-I\right)$, where $\psi_{E}(\lambda)=\left(\psi_{P}(\lambda)-1\right) /(\lambda-1)$. Therefore,

$$
\|(P-I) u\|_{L^{2}} \leqslant C\left\|\left(-h^{2} \Delta-I\right) u\right\|_{L^{2}}
$$

Combining (4.4) and (4.5) we obtain (4.3).

Combining Lemmas 4.1 and 4.2, we obtain the following result.

Lemma 4.3. For all $t \in \mathbb{R}$ and $u \in H^{2}(M)$ we have

$$
\left\|A_{1}(t) u\right\|_{L^{2}} \leqslant C\left\|\mathrm{Op}_{h}(a) u\right\|_{L^{2}}+\frac{C\langle t\rangle}{h}\left\|\left(-h^{2} \Delta-I\right) u\right\|_{L^{2}}+C h\|u\|_{L^{2}},
$$

where $\langle t\rangle:=\sqrt{1+t^{2}}$, and the constant $C$ is independent of $t$ and $h$.

\subsection{Operators corresponding to weighted words}

By Lemma 3.2, for each $\mathbf{w} \in \mathcal{W}\left(N_{0}\right)$ the operator $A_{\mathbf{w}}$ is pseudodifferential modulo an $\mathcal{O}\left(h^{3 / 4}\right)_{L^{2} \rightarrow L^{2}}$ remainder. However, for a subset $\mathcal{E} \subset \mathcal{W}\left(N_{0}\right)$, the operator $A_{\mathcal{E}}$ defined in (3.7) is the sum of many operators of the form $A_{\mathbf{w}}$ and thus a priori might not even be bounded on $L^{2}$ uniformly in $h$. In this section we show that $A_{\mathcal{E}}$ is still a pseudodifferential operator plus a small remainder, using the fact that the corresponding symbol $a_{\mathcal{E}}$ is bounded.

More generally, one can consider operators obtained by assigning a coefficient to each word. For a function $c: \mathcal{W}\left(N_{0}\right) \rightarrow \mathbb{C}$, define the operator $A_{c}$ and the symbol $a_{c}$ by

$$
A_{c}:=\sum_{\mathbf{w} \in \mathcal{W}\left(N_{0}\right)} c(\mathbf{w}) A_{\mathbf{w}} \quad \text { and } \quad a_{c}:=\sum_{\mathbf{w} \in \mathcal{W}\left(N_{0}\right)} c(\mathbf{w}) a_{\mathbf{w}} .
$$

Note that, for $\mathcal{E} \subset \mathcal{W}\left(N_{0}\right)$, we have $A_{\mathcal{E}}=A_{\mathbf{1}_{\mathcal{E}}}$, where $\mathbf{1}_{\mathcal{E}}$ is the indicator function of $\mathcal{E}$.

The next lemma shows that the operator $A_{c}$ is pseudodifferential modulo a small remainder. Recall the symbol classes $S_{L_{s}, \varrho, \varrho^{\prime}}^{\text {comp }}\left(T^{*} M \backslash\{0\}\right)$ introduced in $\S$ A.1.

Lemma 4.4. Assume that $\sup |c| \leqslant 1$. Then,

$$
a_{c} \in S_{L_{s}, 1 / 2,1 / 4}^{\mathrm{comp}}\left(T^{*} M \backslash\{0\}\right) \quad \text { and } \quad A_{c}=\mathrm{Op}_{h}^{L_{s}}\left(a_{c}\right)+\mathcal{O}\left(h^{1 / 2}\right)_{L^{2} \rightarrow L^{2}}
$$

The $S_{L_{s}, 1 / 2,1 / 4}^{\mathrm{comp}}$ seminorms of $a_{c}$ and the constant in $\mathcal{O}\left(h^{1 / 2}\right)$ are independent of $c$.

Proof. We first show that $a_{c} \in S_{L_{s}, 1 / 2,1 / 4}^{\text {comp }}\left(T^{*} M \backslash\{0\}\right)$. As $a_{1}, a_{2} \geqslant 0$ and

$$
a_{1}+a_{2}=1-a_{0} \leqslant 1
$$


for all $(x, \xi) \in T^{*} M \backslash\{0\}$ we have

$$
\left|a_{c}(x, \xi)\right| \leqslant a_{\mathcal{W}\left(N_{0}\right)}(x, \xi)=\prod_{j=0}^{N_{0}-1}\left(a_{1}+a_{2}\right)\left(\varphi_{j}(x, \xi)\right) \leqslant 1 .
$$

It remains to show that, for $m+k>0$ and for all vector fields $Y_{1}, \ldots, Y_{m}, Z_{1}, \ldots, Z_{k}$ on $T^{*} M \backslash\{0\}$ such that $Y_{1}, \ldots, Y_{m}$ are tangent to $L_{s}$, we have

$$
\sup \left|Y_{1} \ldots Y_{m} Z_{1} \ldots Z_{k} a_{c}\right| \leqslant C h^{-k / 2-m / 4}
$$

By the triangle inequality the left-hand side of (4.9) is bounded by

$$
\sum_{\mathbf{w} \in \mathcal{W}\left(N_{0}\right)} \sup \left|Y_{1} \ldots Y_{m} Z_{1} \ldots Z_{k} a_{\mathbf{w}}\right|
$$

By (3.10) each summand is bounded by $C h^{-k / 4-0.01}$, where $C$ is independent of w. The number of summands is equal to $2^{N_{0}} \leqslant h^{-1 / 4+0.01}$. Therefore, the left-hand side of $(4.9)$ is bounded by $C h^{-(k+1) / 4} \leqslant C h^{-k / 2-m / 4}$, giving (4.9).

Finally, by (3.10), we have

$$
A_{c}=\sum_{\mathbf{w} \in \mathcal{W}\left(N_{0}\right)} c(\mathbf{w})\left(\mathrm{Op}_{h}^{L_{s}}\left(a_{\mathbf{w}}\right)+\mathcal{O}\left(h^{3 / 4}\right)_{L^{2} \rightarrow L^{2}}\right)=\mathrm{Op}_{h}^{L_{s}}\left(a_{c}\right)+\mathcal{O}\left(h^{1 / 2}\right)_{L^{2} \rightarrow L^{2}},
$$

finishing the proof.

Combining Lemma 4.4 with the sharp Gårding inequality (Lemma A.4), we deduce the following "almost monotonicity" property for norms of the operators $A_{c}$.

Lemma 4.5. Assume $c, d: \mathcal{W}\left(N_{0}\right) \rightarrow \mathbb{R}$ and $|c(\mathbf{w})| \leqslant d(\mathbf{w}) \leqslant 1$ for all $\mathbf{w} \in \mathcal{W}\left(N_{0}\right)$. Then, for all $u \in L^{2}(M)$, we have

$$
\left\|A_{c} u\right\|_{L^{2}} \leqslant\left\|A_{d} u\right\|_{L^{2}}+C h^{1 / 8}\|u\|_{L^{2}}
$$

where the constant $C$ is independent of $c$ and $d$.

Proof. By (4.8), we may replace $A_{c}$ and $A_{d}$ by $\mathrm{Op}_{h}^{L_{s}}\left(a_{c}\right)$ and $\mathrm{Op}_{h}^{L_{s}}\left(a_{d}\right)$, respectively. It is then enough to prove that

$$
\left\|\mathrm{Op}_{h}^{L_{s}}\left(a_{c}\right) u\right\|_{L^{2}}^{2} \leqslant\left\|\mathrm{Op}_{h}^{L_{s}}\left(a_{d}\right) u\right\|_{L^{2}}^{2}+C h^{1 / 4}\|u\|_{L^{2}}^{2} .
$$

This is equivalent to

$$
\langle B u, u\rangle_{L^{2}} \geqslant-C h^{1 / 4}\|u\|_{L^{2}}^{2}, \quad B:=\mathrm{Op}_{h}^{L_{s}}\left(a_{d}\right)^{*} \mathrm{Op}_{h}^{L_{s}}\left(a_{d}\right)-\mathrm{Op}_{h}^{L_{s}}\left(a_{c}\right)^{*} \mathrm{Op}_{h}^{L_{s}}\left(a_{c}\right)
$$


Recall that $a_{c}, a_{d} \in S_{L_{s}, 1 / 2,1 / 4}^{\mathrm{comp}}\left(T^{*} M \backslash\{0\}\right)$. By (A.23) and (A.24), we have

$$
B=\operatorname{Op}_{h}^{L_{s}}\left(a_{d}^{2}-a_{c}^{2}\right)+\mathcal{O}\left(h^{1 / 4}\right)_{L^{2} \rightarrow L^{2}} .
$$

As $|c(\mathbf{w})| \leqslant d(\mathbf{w})$ for all $\mathbf{w}$, we have

$$
0 \leqslant a_{d}^{2}-a_{c}^{2} \in S_{L_{s}, 1 / 2,1 / 4}^{\mathrm{comp}}\left(T^{*} M \backslash\{0\}\right) .
$$

Then, by Lemma A.4,

$$
\operatorname{Re}\left\langle\mathrm{Op}_{h}^{L_{s}}\left(a_{d}^{2}-a_{c}^{2}\right) u, u\right\rangle_{L^{2}} \geqslant-C h^{1 / 4}\|u\|_{L^{2}}^{2} .
$$

Combining (4.11) and (4.12), we get (4.10), finishing the proof.

\subsection{Proof of Proposition 3.4}

We first estimate $A_{\mathcal{Z}} u$, where $\mathcal{Z} \subset \mathcal{W}\left(N_{0}\right)$ is the set of controlled words defined in (3.15).

Lemma 4.6. For all $u \in H^{2}(M)$, with the constant $C$ independent of $\alpha$,

$$
\left\|A_{\mathcal{Z}} u\right\|_{L^{2}} \leqslant \frac{C}{\alpha}\left\|\mathrm{Op}_{h}(a) u\right\|_{L^{2}}+\frac{C \log (1 / h)}{\alpha h}\left\|\left(-h^{2} \Delta-I\right) u\right\|_{L^{2}}+\mathcal{O}\left(h^{1 / 8}\right)\|u\|_{L^{2}}
$$

Proof. Recall the density function $F$ from (3.14). By definition, the indicator function $\mathbf{1}_{\mathcal{Z}}$ satisfies $0 \leqslant \alpha \mathbf{1}_{\mathcal{Z}} \leqslant F \leqslant 1$. Thus, by Lemma 4.5 (where $A_{F}$ is defined by (4.7)),

$$
\alpha\left\|A_{\mathcal{Z}} u\right\|_{L^{2}} \leqslant\left\|A_{F} u\right\|_{L^{2}}+\mathcal{O}\left(h^{1 / 8}\right)\|u\|_{L^{2}} .
$$

Using the definition (3.14) together with (3.8), we rewrite $A_{F}$ as follows:

$$
A_{F}=\frac{1}{N_{0}} \sum_{j=0}^{N_{0}-1} \sum_{\substack{\mathbf{w} \in \mathcal{W}\left(N_{0}\right) \\ w_{j}=1}} A_{\mathbf{w}}=\frac{1}{N_{0}} \sum_{j=0}^{N_{0}-1}\left(A_{1}+A_{2}\right)^{N_{0}-1-j} A_{1}(j)\left(A_{1}+A_{2}\right)^{j} .
$$

Recall that $\left\|A_{1}+A_{2}\right\|_{L^{2} \rightarrow L^{2}} \leqslant 1$; see the proof of Lemma 3.1. Then,

$$
\left\|A_{F} u\right\|_{L^{2}} \leqslant \max _{0 \leqslant j<N_{0}}\left\|A_{1}(j)\left(A_{1}+A_{2}\right)^{j} u\right\|_{L^{2}} .
$$

Since $\left\|A_{1}(j)\right\|_{L^{2} \rightarrow L^{2}}=\left\|A_{1}\right\|_{L^{2} \rightarrow L^{2}} \leqslant C$ and using the estimate of $\left(A_{1}+A_{2}\right)^{j} u-u$ given in Lemma 3.1, we get

$$
\left\|A_{F} u\right\|_{L^{2}} \leqslant \max _{0 \leqslant j<N_{0}}\left\|A_{1}(j) u\right\|_{L^{2}}+C\left\|\left(-h^{2} \Delta-I\right) u\right\|_{L^{2}} .
$$

Estimating $A_{1}(j) u$ by Lemma 4.3 , we get

$$
\left\|A_{F} u\right\|_{L^{2}} \leqslant C\left\|\mathrm{Op}_{h}(a) u\right\|_{L^{2}}+\frac{C \log (1 / h)}{h}\left\|\left(-h^{2} \Delta-I\right) u\right\|_{L^{2}}+\mathcal{O}(h)\|u\|_{L^{2}} .
$$

Combining (4.14) and (4.15), we obtain (4.13). 
We now finish the proof of Proposition 3.4. Recalling (3.16), we write

$$
\mathcal{Y}=\bigsqcup_{\ell=1}^{8} \mathcal{Y}_{\ell}, \quad \mathcal{Y}_{\ell}:=\left\{\mathbf{w}^{(1)} \ldots \mathbf{w}^{(8)}: \mathbf{w}^{(\ell)} \in \mathcal{Z} \text { and } \mathbf{w}^{(\ell+1)}, \ldots, \mathbf{w}^{(8)} \in \mathcal{W}\left(N_{0}\right) \backslash \mathcal{Z}\right\}
$$

Then,

$$
A_{\mathcal{Y}}=\sum_{\ell=1}^{8} A_{\mathcal{Y}_{\ell}}
$$

Let $\mathcal{Q}:=\mathcal{W}\left(N_{0}\right) \backslash \mathcal{Z}$. Then, using (3.8), we have the following factorization:

$$
A_{\mathcal{Y}_{\ell}}=A_{\mathcal{Q}}\left(7 N_{0}\right) \ldots A_{\mathcal{Q}}\left(\ell N_{0}\right) A_{\mathcal{Z}}\left((\ell-1) N_{0}\right)\left(A_{1}+A_{2}\right)^{(\ell-1) N_{0}} .
$$

By Lemma 4.4 , we have $\left\|A_{\mathcal{Q}}\right\|_{L^{2} \rightarrow L^{2}},\left\|A_{\mathcal{Z}}\right\|_{L^{2} \rightarrow L^{2}} \leqslant C$. Estimating

$$
\left(A_{1}+A_{2}\right)^{(\ell-1) N_{0}} u-u
$$

by Lemma 3.1, we get

$$
\left\|A_{\mathcal{Y}} u\right\|_{L^{2}} \leqslant C \sum_{\ell=1}^{8}\left\|A_{\mathcal{Z}}\left((\ell-1) N_{0}\right) u\right\|_{L^{2}}+C\left\|\left(-h^{2} \Delta-I\right) u\right\|_{L^{2}} .
$$

By Lemma 4.2, we have

$$
\left\|A_{\mathcal{Z}}\left((\ell-1) N_{0}\right) u\right\|_{L^{2}} \leqslant\left\|A_{\mathcal{Z}} u\right\|_{L^{2}}+\frac{C \log (1 / h)}{h}\left\|\left(-h^{2} \Delta-I\right) u\right\|_{L^{2}}
$$

Using Lemma 4.6 to bound $\left\|A_{\mathcal{Z}} u\right\|_{L^{2}}$ and combining (4.16) with (4.17), we obtain (3.18), finishing the proof.

\section{Fractal uncertainty principle}

In this section we prove Proposition 3.5 using the fractal uncertainty principle established in $[\mathrm{BD}]$.

\subsection{Fractal uncertainty principle for porous sets in $\mathbb{R}$}

We start by adapting the result of $[\mathrm{BD}]$ to the setting of porous sets, by embedding them into Ahlfors-David regular sets of some dimension $\delta<1$. Here, we define porous sets as follows. 
Definition 5.1. Let $\nu \in(0,1)$ and $0<\alpha_{0} \leqslant \alpha_{1}$. We say that a subset $\Omega$ of $\mathbb{R}$ is $\nu$-porous on scales $\alpha_{0}$ to $\alpha_{1}$ if for each interval $I$ of size $|I| \in\left[\alpha_{0}, \alpha_{1}\right]$, there exists a subinterval $J \subset I$ with $|J|=\nu|I|$ such that $J \cap \Omega=\varnothing$.

As for Ahlfors-David regular sets, we recall the following definition.

Definition 5.2. [BD, Definition 1.1] Let $\delta \in[0,1], C_{R} \geqslant 1$, and $0 \leqslant \alpha_{0} \leqslant \alpha_{1}$. We say that a closed non-empty subset $X$ of $\mathbb{R}$ is $\delta$-regular with constant $C_{R}$ on scales $\alpha_{0}$ to $\alpha_{1}$ if there exists a Borel measure $\mu_{X}$ on $\mathbb{R}$ such that

(1) $\mu_{X}$ is supported on $X$, that is $\mu_{X}(\mathbb{R} \backslash X)=0$;

(2) for any interval $I$ with $\alpha_{0} \leqslant|I| \leqslant \alpha_{1}$, we have $\mu_{X}(I) \leqslant C_{R}|I|^{\delta}$;

(3) if in addition $I$ is centered at a point in $X$, then $\mu_{X}(I) \geqslant C_{R}^{-1}|I|^{\delta}$.

We use the following version of fractal uncertainty principle for $\delta$-regular sets. Henceforth, for $X \subset \mathbb{R}$ and $s>0, X(s)=X+[-s, s]$ denotes the $s$-neighborhood of $X$.

Proposition 5.3. ([BD, Proposition 4.3]) Let $B=B(h): L^{2}(\mathbb{R}) \rightarrow L^{2}(\mathbb{R})$ be defined as

$$
B f(x)=h^{-1 / 2} \int e^{i \Phi(x, y) / h} b(x, y) f(y) d y,
$$

where $\Phi \in C^{\infty}(U ; \mathbb{R}), b \in C_{0}^{\infty}(U), U \subset \mathbb{R}^{2}$ is open, and $\partial_{x y}^{2} \Phi \neq 0$ on $U$.

Let $0 \leqslant \delta<1$ and $C_{R} \geqslant 1$. Then, there exist $\beta>0, \varrho \in(0,1)$ depending only on $\delta$ and $C_{R}$, and there exists $C>0$ depending only on $\delta, C_{R}, b$ and $\Phi$ such that, for all $h \in(0,1)$ and all $X, Y \subset \mathbb{R}$ which are $\delta$-regular with constant $C_{R}$ on scales 0 to 1 ,

$$
\left\|\mathbb{1}_{X\left(h^{\varrho}\right)} B(h) \mathbb{1}_{Y\left(h^{\varrho}\right)}\right\|_{L^{2}(\mathbb{R}) \rightarrow L^{2}(\mathbb{R})} \leqslant C h^{\beta} .
$$

Although porous sets need not be regular, we can always embed a porous set $\Omega$ in a neighborhood of a $\delta$-regular set $X$ with $\delta<1$. The set $X$ is constructed by a Cantor-like procedure with some large base $L$, where at the $k$ th step we remove intervals of size $L^{-k-1}$, which do not intersect $\Omega$.

Lemma 5.4. For each $\nu \in(0,1)$ there exist $\delta=\delta(\nu) \in(0,1)$ and $C_{R}=C_{R}(\nu) \geqslant 1$ such that the following holds. Let $\Omega$ be a $\nu$-porous set on scales $\alpha_{0}$ to 1 . Then, there exists a set $X$ which is $\delta$-regular with constant $C_{R}$ on scales 0 to 1 such that $\Omega \subset X\left(\alpha_{0}\right)$.

Proof. Put $L:=\lceil 2 / \nu\rceil \in \mathbb{N}$. We use the tree of intervals

$$
I_{m, k}=\left[m L^{-k},(m+1) L^{-k}\right], \quad m, k \in \mathbb{Z} .
$$

Let $k_{0} \geqslant 0$ be the unique integer such that $L^{-1-k_{0}}<\alpha_{0} \leqslant L^{-k_{0}}$.

Take $m$ and $k$ with $0 \leqslant k \leqslant k_{0}$. We claim that there exists $n=n(m, k)$ such that

$$
I_{n, k+1} \subset I_{m, k}, \quad I_{n, k+1} \cap \Omega=\varnothing .
$$


Indeed, as $\Omega$ is $\nu$-porous, there is a subinterval $J \subset I_{m, k}$ such that $|J|=\nu\left|I_{m, k}\right| \geqslant 2 L^{-k-1}$ and $J \cap \Omega=\varnothing$. Then, one can find $n$ such that $I_{n, k+1} \subset J$, and this value of $n$ satisfies (5.3). When $k>k_{0}$, we put $n(m, k):=L m$, so that the condition $I_{n(m, k), k+1} \subset I_{m, k}$ still holds.

We now define the set $X$ as follows:

$$
X:=\bigcap_{k=0}^{\infty} \bar{X}_{k}, \quad X_{k}:=\mathbb{R} \backslash \bigcup_{m \in \mathbb{Z}} I_{n(m, k), k+1} .
$$

Note that, for each $k \geqslant 1$, there exists a set $\mathcal{M}(k) \subset \mathbb{Z}$ such that

$$
\bigcap_{\ell=0}^{k-1} \bar{X}_{\ell}=\bigcup_{m \in \mathcal{M}(k)} I_{m, k}
$$

We set $\mathcal{M}(0):=\mathbb{Z}$. Then, for all $k \geqslant 0$ and $m$, we have

$$
\#\left\{m^{\prime} \in \mathcal{M}(k+1): I_{m^{\prime}, k+1} \subset I_{m, k}\right\}= \begin{cases}L-1, & m \in \mathcal{M}(k), \\ 0, & \text { otherwise }\end{cases}
$$

We claim that $\Omega \subset X\left(\alpha_{0}\right)$. Indeed, by (5.3), we have $\Omega \subset X_{k}$ when $0 \leqslant k \leqslant k_{0}$. Take $x \in \Omega$. Then, $x$ lies in $\bigcap_{k=0}^{k_{0}} \bar{X}_{k}$, which implies that $x \in I_{m, k_{0}+1}$ for some $m \in \mathcal{M}\left(k_{0}+1\right)$. Since $L \geqslant 2$, by induction using (5.4), there exists a sequence $\left(m_{k} \in \mathcal{M}(k)\right)_{k \geqslant k_{0}+1}$ with $m_{k_{0}+1}=$ $m$ and $I_{m_{k+1}, k+1} \subset I_{m_{k}, k}$. The intersection $\bigcap_{k} I_{m_{k}, k}$ consists of a single point $y \in X$. Since $x, y \in I_{m, k_{0}+1}$, we have $|x-y| \leqslant L^{-k_{0}-1}$, and thus $x \in X\left(L^{-k_{0}-1}\right) \subset X\left(\alpha_{0}\right)$ as required.

It remains to prove that $X$ is $\delta$-regular with some constant $C_{R}$ on scales 0 to 1 , where we put

$$
\delta:=\frac{\log (L-1)}{\log L} \in(0,1)
$$

Let $\mu_{X}$ be the natural Cantor-like measure supported on $X$. More precisely, by (5.4) there exists a unique Borel measure $\mu_{X}$ on $\mathbb{R}$ satisfying, for all $m$ and $k \geqslant 0$,

$$
\mu_{X}\left(I_{m, k}\right)= \begin{cases}(L-1)^{-k}=L^{-\delta k}, & m \in \mathcal{M}(k), \\ 0, & \text { otherwise }\end{cases}
$$

Take an interval $I$ of size $|I| \leqslant 1$, and fix the unique integer $k \geqslant 0$ such that

$$
L^{-k-1}<|I| \leqslant L^{-k}
$$

Then, there exists $m$ such that $I \subset I_{m, k} \cup I_{m+1, k}$. It follows that

$$
\mu_{X}(I) \leqslant \mu_{X}\left(I_{m, k}\right)+\mu_{X}\left(I_{m+1, k}\right) \leqslant 2 L^{-\delta k} \leqslant 2 L|I|^{\delta} .
$$


Next, assume that $I$ is an interval of size $|I| \leqslant 1$ centered at a point $x \in X$. Fix the unique integer $k \geqslant 0$ such that $2 L^{-k-1} \leqslant|I|<2 L^{-k}$, and choose $m \in \mathcal{M}(k+1)$ such that $x \in I_{m, k+1}$. Then $I_{m, k+1} \subset I$, and thus

$$
\mu_{X}(I) \geqslant \mu_{X}\left(I_{m, k+1}\right)=L^{-\delta(k+1)} \geqslant \frac{|I|^{\delta}}{2 L} .
$$

Recalling Definition 5.2, we see that (5.5) and (5.6) imply that $X$ is $\delta$-regular with constant $C_{R}:=2 L$ on scales 0 to 1 . This finishes the proof.

Combining Proposition 5.3 and Lemma 5.4, we obtain the following fractal uncertainty principle for $\nu$-porous sets.

Proposition 5.5. Let $K>0$ and $\nu \in(0,1)$ be fixed and $B(h): L^{2}(\mathbb{R}) \rightarrow L^{2}(\mathbb{R})$ be as in Proposition 5.3. Then, there exist $\beta>0$ and $\varrho \in(0,1)$ depending only on $\nu$, and there exists $C$ depending only on $\nu, K, b$, and $\Phi$ such that, for all $h \in(0,1)$ and all $\Omega_{ \pm} \subset \mathbb{R}$ which are $\nu$-porous on scales $K h^{\varrho}$ to 1 ,

$$
\left\|\mathbb{1}_{\Omega_{-}\left(K h^{\varrho}\right)} B(h) \mathbb{1}_{\Omega_{+}\left(K h^{\varrho}\right)}\right\|_{L^{2} \rightarrow L^{2}} \leqslant C h^{\beta} .
$$

Proof. By Lemma 5.4, there exist $X, Y \subset \mathbb{R}$ which are $\delta$-regular with constant $C_{R}$ on scales 0 to 1 for some $\delta=\delta(\nu) \in(0,1), C_{R}=C_{R}(\nu)$, such that

$$
\Omega_{-} \subset X\left(K h^{\varrho}\right) \text { and } \Omega_{+} \subset Y\left(K h^{\varrho}\right) .
$$

Then,

$$
\left\|\mathbb{1}_{\Omega_{-}\left(K h^{\varrho}\right)} B(h) \mathbb{1}_{\Omega_{+}\left(K h^{\varrho}\right)}\right\|_{L^{2} \rightarrow L^{2}} \leqslant\left\|\mathbb{1}_{X\left(2 K h^{\varrho}\right)} B(h) \mathbb{1}_{Y\left(2 K h^{\varrho}\right)}\right\|_{L^{2} \rightarrow L^{2}} .
$$

It remains to apply Proposition 5.3, where we increase $\varrho$ slightly to absorb the constant $2 K$.

\subsection{Fractal uncertainty principle for porous sets in $T^{*} M$}

We use Proposition 5.5 to prove a fractal uncertainty principle for subsets of $T^{*} M \backslash\{0\}$, where $M$ is a compact orientable hyperbolic surface.

Let $H_{p}, U_{+}, U_{-}, D$ be the frame on $T^{*} M \backslash\{0\}$ defined in (2.3). For $v=\left(v_{1}, v_{2}, v_{3}\right) \in$ $\mathbb{R}^{3}$, define the vector fields

$$
\mathcal{V}_{ \pm} v=v_{1} H_{p}+v_{2} D+v_{3} U_{ \pm} .
$$

For $(x, \xi) \in T^{*} M \backslash\{0\}$ and $\nu_{0}, \nu_{1}>0$, we define the stable $\left(\nu_{0}, \nu_{1}\right)$ slice centered at $(x, \xi)$ as follows:

$$
\Sigma_{\nu_{0}, \nu_{1}}^{+}(x, \xi):=\left\{e^{\mathcal{V}_{-} v} e^{s U_{+}}(x, \xi):|s| \leqslant \nu_{0} \text { and }|v| \leqslant \nu_{1}\right\} .
$$

Similarly define the unstable $\left(\nu_{0}, \nu_{1}\right)$ slice centered at $(x, \xi)$ :

$$
\Sigma_{\nu_{0}, \nu_{1}}^{-}(x, \xi):=\left\{e^{\mathcal{V}_{+} v} e^{s U_{-}}(x, \xi):|s| \leqslant \nu_{0} \text { and }|v| \leqslant \nu_{1}\right\}
$$




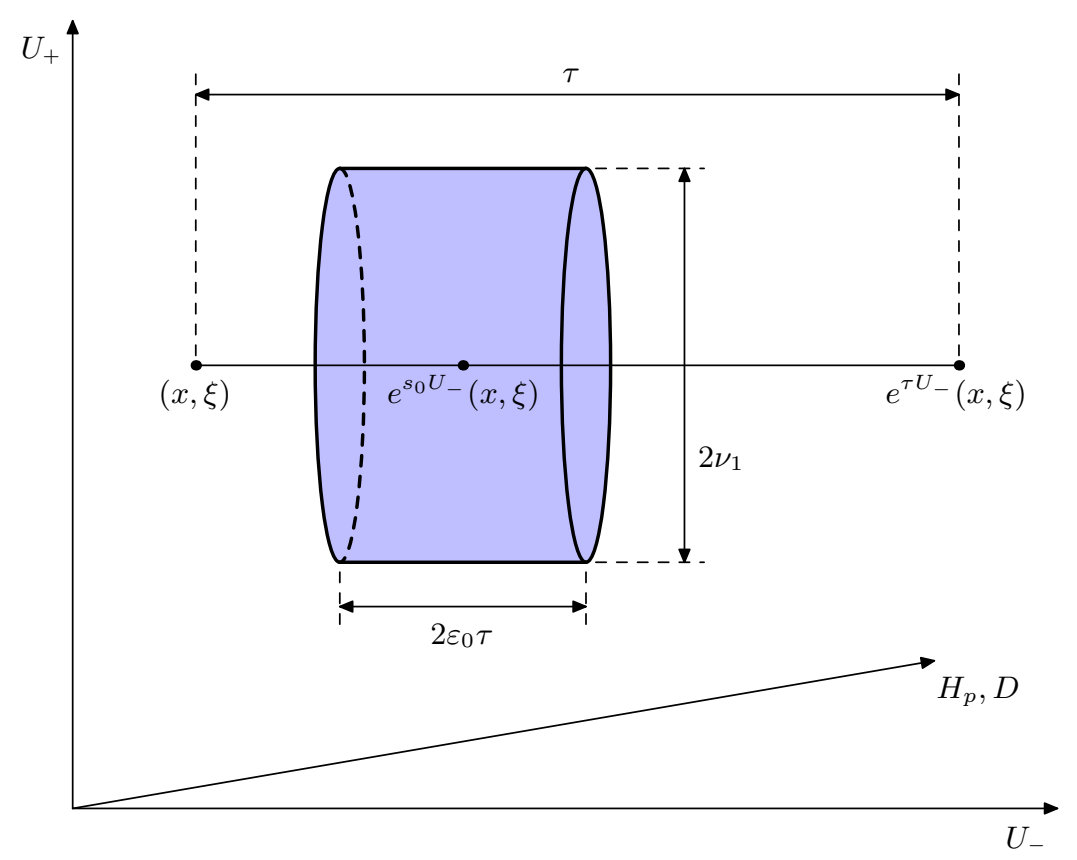

Figure 2. An illustration of Definition 5.6 of an $\left(\varepsilon_{0}, \nu_{1}\right)$-porous set along $U_{-}$. The blue cylinder is the unstable slice $\Sigma_{\varepsilon_{0} \tau, \nu_{1}}^{-}\left(e^{s_{0} U_{-}}(x, \xi)\right)$. (We ignore here the fact that $H_{p}, U_{ \pm}$, and $D$ do not commute, and thus do not give rise to a coordinate system.)

Definition 5.6. Let

$$
Z \subset\left\{\frac{1}{4} \leqslant|\xi|_{g} \leqslant 4\right\} \subset T^{*} M \backslash\{0\}
$$

be a closed set and fix $\varepsilon_{0}, \nu_{1}, \tau_{0} \in(0,1]$. We say that $Z$ is $\left(\varepsilon_{0}, \nu_{1}\right)$-porous along $U_{ \pm}$up to scale $\tau_{0}$ if, for each $(x, \xi) \in T^{*} M \backslash\{0\}$ and each $\tau \in\left[\tau_{0}, 1\right]$, there exists $s_{0} \in[0, \tau]$ such that (see Figure 2)

$$
\Sigma_{\varepsilon_{0} \tau, \nu_{1}}^{ \pm}\left(e^{s_{0} U_{ \pm}}(x, \xi)\right) \cap Z=\varnothing .
$$

Our fractal uncertainty principle for subsets of $T^{*} M \backslash\{0\}$ is formulated in terms of the $\Psi_{h, L, \varrho}^{\text {comp }}\left(T^{*} M \backslash\{0\}\right)$ calculus introduced in $\S 2.3$.

Proposition 5.7. Fix $\varepsilon_{0}, \nu_{1} \in(0,1]$. Then, there exist $\beta>0$ and $\varrho \in(0,1)$ depending only on $M, \varepsilon_{0}$, and $\nu_{1}$ such that the following holds. Suppose that

$$
a_{+} \in S_{L_{u}, \varrho}^{\mathrm{comp}}\left(T^{*} M \backslash\{0\}\right) \quad \text { and } \quad a_{-} \in S_{L_{s}, \varrho}^{\mathrm{comp}}\left(T^{*} M \backslash\{0\}\right),
$$

and $\operatorname{supp} a_{ \pm} i s\left(\varepsilon_{0}, \nu_{1}\right)$-porous along $U_{ \pm}$, up to scale $K_{1} h^{\varrho}$ for some constant $K_{1}$. Then, for all $Q \in \Psi_{h}^{0}(M)$,

$$
\left\|\mathrm{Op}_{h}^{L_{s}}\left(a_{-}\right) Q \mathrm{Op}_{h}^{L_{u}}\left(a_{+}\right)\right\|_{L^{2} \rightarrow L^{2}} \leqslant C h^{\beta},
$$

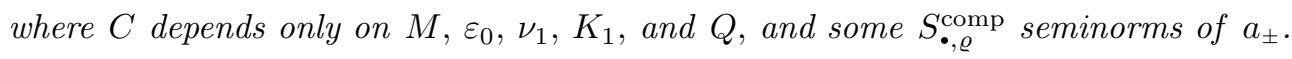


In the rest of this subsection, we prove Proposition 5.7. We begin by straightening out weak stable/unstable Lagrangian foliations similarly to [DZa, §4.4]. Denote by $\mathbb{H}^{2}$ the hyperbolic plane; it is the universal cover of $M$. Let

$$
\begin{aligned}
\varkappa^{ \pm}: T^{*} \mathbb{H}^{2} \backslash\{0\} & \longrightarrow T^{*}\left(\mathbb{R}_{w}^{+} \times \mathbb{S}_{y}^{1}\right), \\
(x, \xi) & \longmapsto(w, y, \theta, \eta),
\end{aligned}
$$

be the exact symplectomorphisms constructed in [DZa, Lemma 4.7] mapping $L_{s}$ and $L_{u}$ to the vertical foliation $L_{0}$ on $T^{*}\left(\mathbb{R}^{+} \times \mathbb{S}^{1}\right)$ :

$$
\left(\varkappa^{+}\right)_{*} L_{u}=\left(\varkappa^{-}\right)_{*} L_{s}=L_{0}=\operatorname{ker}(d w) \cap \operatorname{ker}(d y) .
$$

More precisely, in the Poincaré disk model of $\mathbb{H}^{2}$, we have $w=p(x, \xi)=|\xi|_{g}$,

$$
y=B_{\mp}(x, \xi)
$$

is the limit of the projection to $\mathbb{H}^{2}$ of the geodesic $e^{t H_{p}}(x, \xi)$ as $t \rightarrow \mp \infty$ on the boundary $\mathbb{S}^{1}=\partial \mathbb{H}^{2}$,

$$
\theta= \pm \log \mathcal{P}\left(x, B_{\mp}(x, \xi)\right),
$$

where

$$
\mathcal{P}(x, y)=\frac{1-|x|^{2}}{|x-y|^{2}}, \quad x \in \mathbb{H}^{2} \text { and } y \in \mathbb{S}^{1},
$$

is the Poisson kernel, and

$$
\eta= \pm G_{\mp}(x, \xi)= \pm p(x, \xi) \mathcal{G}\left(B_{\mp}(x, \xi), B_{ \pm}(x, \xi)\right) \in T_{B_{\mp}(x, \xi)}^{*} \mathbb{S}^{1}
$$

where (see [DZa, formula (1.19)])

$$
\mathcal{G}\left(y, y^{\prime}\right)=\frac{y^{\prime}-\left(y \cdot y^{\prime}\right) y}{1-y \cdot y^{\prime}} \in T_{y}^{*} \mathbb{S}^{1} \simeq T_{y} \mathbb{S}^{1} \subset \mathbb{R}^{2}, \quad y, y^{\prime} \in \mathbb{S}^{1}, y \neq y^{\prime}
$$

is half the stereographic projection of $y^{\prime}$ with base $y$. See Figure 3 .

It follows from the definition of $B_{ \pm}(x, \xi)$ that

$$
\left(\mathcal{V}_{ \pm} v\right) B_{ \pm}=0 \quad \text { for all } v \in \mathbb{R}^{3}
$$

By a microlocal partition of unity and since supp $a_{ \pm} \subset\left\{\frac{1}{4} \leqslant|\xi|_{g} \leqslant 4\right\}$, we may assume that $\mathrm{WF}_{h}(Q) \subset V$, where $V$ is a sufficiently small neighborhood of any given point $\left(x_{0}, \xi_{0}\right) \in$ $T^{*} M \backslash\{0\}$. We assume that

$$
\operatorname{diam}(V) \leqslant \frac{\nu_{1}}{C_{0}},
$$




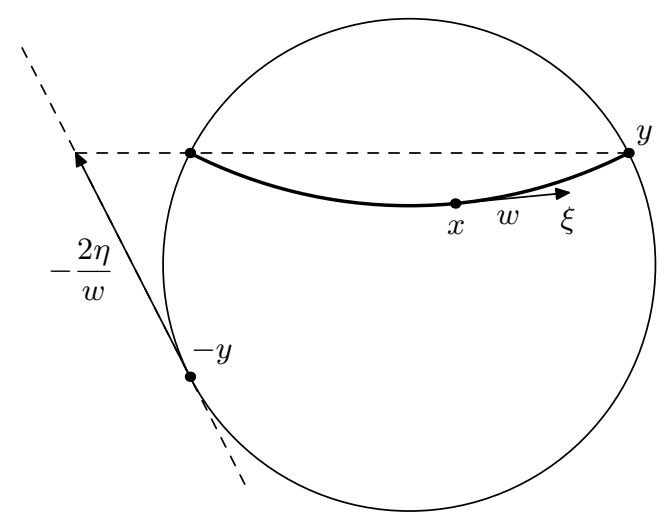

Figure 3. The coordinates $(w, y, \theta, \eta)=\varkappa_{-}(x, \xi)$ in the Poincaré disk model of $\mathbb{H}^{2}$. Here, $w$ is the length of $\xi, y$ is the limit of the geodesic starting from $(x, \xi)$ at $t \rightarrow \infty, \theta$ is determined from the Poisson kernel $\mathcal{P}(x, y)$, and $\eta$ is determined from the stereographic projection pictured. By (5.9), the value of $y$ does not change if we deform $(x, \xi)$ along the stable, flow, or dilation direction.

where $C_{0}$ is a large constant depending only on $M$ to be chosen in Lemma 5.8 below. We lift $V \subset T^{*} M \backslash\{0\}$ to a subset of $T^{*} \mathbb{H}^{2} \backslash 0$ and use $\varkappa^{ \pm}$to define the symplectomorphisms onto their images

$$
\varkappa_{0}^{ \pm}: V \longrightarrow T^{*}\left(\mathbb{R}^{+} \times \mathbb{S}^{1}\right) .
$$

Note that we can make $\varkappa_{0}^{ \pm}(V)$ contained in a compact subset of $T^{*}\left(\mathbb{R}^{+} \times \mathbb{S}^{1}\right)$ which only depends on $M$.

We next quantize $\varkappa_{0}^{ \pm}$by Fourier integral operators which conjugate $\mathrm{Op}_{h}^{L_{s}}\left(a_{-}\right)$and $\mathrm{Op}_{h}^{L_{u}}\left(a_{+}\right)$to operators on $\mathbb{R}^{+} \times \mathbb{S}^{1}$. Following [DZa, $\S 4.4$, proof of Theorem 3], we consider operators

$$
\mathcal{B}_{ \pm} \in I_{h}^{\mathrm{comp}}\left(\varkappa_{0}^{ \pm}\right) \quad \text { and } \quad \mathcal{B}_{ \pm}^{\prime} \in I_{h}^{\mathrm{comp}}\left(\left(\varkappa_{0}^{ \pm}\right)^{-1}\right)
$$

quantizing $\varkappa_{0}^{ \pm}$near $\varkappa_{0}^{ \pm}\left(\mathrm{WF}_{h}(Q)\right) \times \mathrm{WF}_{h}(Q)$ in the sense of (A.16). Consider the following operators on $L^{2}\left(\mathbb{R}^{+} \times \mathbb{S}^{1}\right)$ :

$$
A_{-}:=\mathcal{B}_{-} \mathrm{Op}_{h}^{L_{s}}\left(a_{-}\right) \mathcal{B}_{-}^{\prime}, \quad A_{+}:=\mathcal{B}_{+} Q \mathrm{Op}_{h}^{L_{u}}\left(a_{+}\right) \mathcal{B}_{+}^{\prime}, \quad \text { and } \quad B=\mathcal{B}_{-} \mathcal{B}_{+}^{\prime} .
$$

Then, similarly to [DZa, formula (4.58)],

$$
\mathrm{Op}_{h}^{L_{s}}\left(a_{-}\right) Q \mathrm{Op}_{h}^{L_{u}}\left(a_{+}\right)=\mathcal{B}_{-}^{\prime} A_{-} B A_{+} \mathcal{B}_{+}+\mathcal{O}\left(h^{\infty}\right)_{L^{2} \rightarrow L^{2}}
$$

Moreover, by (A.22), there exist $\tilde{a}_{ \pm} \in S_{L_{0}, \varrho}^{\text {comp }}\left(T^{*}\left(\mathbb{R}^{+} \times \mathbb{S}^{1}\right)\right)$ such that

$$
A_{ \pm}=\mathrm{Op}_{h}^{L_{0}}\left(\tilde{a}_{ \pm}\right)+\mathcal{O}\left(h^{\infty}\right)_{L^{2} \rightarrow L^{2}}, \quad \text { and } \quad \operatorname{supp} \tilde{a}_{ \pm} \subset \varkappa_{0}^{ \pm}\left(V \cap \operatorname{supp} a_{ \pm}\right) .
$$

Therefore, in order to establish (5.8), it suffices to prove that

$$
\left\|\mathrm{Op}_{h}^{L_{0}}\left(\tilde{a}_{-}\right) B \mathrm{Op}_{h}^{L_{0}}\left(\tilde{a}_{+}\right)\right\|_{L^{2}\left(\mathbb{R}^{+} \times \mathbb{S}^{1}\right) \rightarrow L^{2}\left(\mathbb{R}^{+} \times \mathbb{S}^{1}\right)} \leqslant C h^{\beta} .
$$


Using the porosity of $\operatorname{supp} a_{ \pm}$along $U_{ \pm}$, we get the following 1-dimensional porosity statement for projections of $\operatorname{supp} \tilde{a}_{ \pm}$.

Lemma 5.8. There exists a constant $C_{0}>0$, depending only on $M$, such that the following holds. Define the projections of supp $\tilde{a}_{ \pm}$onto the $y$ variable

$$
\Omega_{ \pm}:=\left\{y \in \mathbb{S}^{1}:(w, y, \theta, \eta) \in \operatorname{supp} \tilde{a}_{ \pm} \text {for some } w, \theta \text { and } \eta\right\} \subset \mathbb{S}^{1}
$$

Then, $\Omega_{ \pm}$(more precisely, their lifts to $\mathbb{R}$ ) are $\nu$-porous on scales $\alpha_{0}$ to 1 in the sense of Definition 5.1, where $\nu:=\varepsilon_{0} \nu_{1} / C_{0}$ and $\alpha_{0}:=C_{0} \nu_{1}^{-1} K_{1} h^{\varrho}$.

Proof. We show the porosity of $\Omega_{+}$, with the case of $\Omega_{-}$handled similarly. Denote by $C_{1}>0$ a large constant depending only on $M$ and put $C_{0}:=C_{1}^{4}$.

Denote $W:=\varkappa_{0}^{+}(V)$. Let $V^{\prime}$ be the $\left(\nu_{1} / C_{1}^{2}\right)$-neighborhood of $V$ and $V^{\prime \prime}$ be the $\left(\nu_{1} / C_{1}^{2}\right)$-neighborhood of $V^{\prime}$. Lifting $V^{\prime \prime}$ to $T^{*} \mathbb{H}^{2} \backslash\{0\}$ and using $\varkappa^{+}$, we extend $\varkappa_{0}^{+}$to a symplectomorphism

$$
\varkappa_{0}^{+}: V^{\prime} \longrightarrow W^{\prime}, V^{\prime \prime} \longrightarrow W^{\prime \prime}
$$

for some open sets $W^{\prime}, W^{\prime \prime} \subset T^{*}\left(\mathbb{R}^{+} \times \mathbb{S}^{1}\right)$. Note that, by (5.10),

$$
\operatorname{diam}\left(W^{\prime \prime}\right) \leqslant \frac{C_{1}}{10} \operatorname{diam}\left(V^{\prime \prime}\right) \leqslant \frac{\nu_{1}}{C_{1}} .
$$

Moreover, the $\left(\nu_{1} / C_{1}^{3}\right)$-neighborhoods of $W$ and $W^{\prime}$ are contained in $W^{\prime}$ and $W^{\prime \prime}$ respectively.

Let $I \subset \mathbb{S}^{1}$ be an interval with $\alpha_{0} \leqslant|I| \leqslant 1$ centered at some $y_{0} \in \mathbb{S}^{1}$. Assume first that the $y$-projection of $W^{\prime}$ does not contain $y_{0}$. Then, since $\operatorname{supp} \tilde{a}_{+} \subset W$ by (5.11), we see that $y_{0}$ lies distance at least $\nu_{1} / C_{0}$ away from $\Omega_{+}$. Thus, the interval of size $\nu|I|$ centered at $y_{0}$ does not intersect $\Omega_{+}$and verifies the porosity condition in Definition 5.1.

We henceforth assume that the $y$-projection of $W^{\prime}$ does contain $y_{0}$. Choose $w_{0}, \theta_{0}$ and $\eta_{0}$ such that $\left(w_{0}, y_{0}, \theta_{0}, \eta_{0}\right) \in W^{\prime}$. Let $\left(x_{0}, \xi_{0}\right):=\left(\varkappa_{0}^{+}\right)^{-1}\left(w_{0}, y_{0}, \theta_{0}, \eta_{0}\right) \in V^{\prime}$. Put

$$
\tau:=C_{1}^{-3} \nu_{1}|I| \quad \text { and } \quad K_{1} h^{\varrho} \leqslant \tau \leqslant \frac{\nu_{1}}{C_{1}^{3}} \leqslant 1 .
$$

Since supp $a_{+}$is $\left(\varepsilon_{0}, \nu_{1}\right)$-porous along $U_{+}$up to scale $K_{1} h^{\varrho}$, there exists $s_{0} \in[0, \tau]$ such that

$$
\Sigma_{\varepsilon_{0} \tau, \nu_{1}}^{+}\left(x_{1}, \xi_{1}\right) \cap \operatorname{supp} a_{+}=\varnothing, \quad \text { where }\left(x_{1}, \xi_{1}\right):=e^{s_{0} U_{+}}\left(x_{0}, \xi_{0}\right) \in V^{\prime \prime} .
$$

Since $C_{1}$ is large and $H_{p}, U_{+}, U_{-}$and $D$ form a frame, we have a diffeomorphism

$$
\begin{aligned}
& \Theta: \widetilde{U} \longrightarrow W^{\prime \prime}, \\
& (s, v) \longmapsto \varkappa_{0}^{+}\left(e^{\mathcal{V}_{-} v} e^{s U_{+}}\left(x_{1}, \xi_{1}\right)\right),
\end{aligned}
$$


where $\widetilde{U}$ is some neighborhood of $(0,0)$ in $\mathbb{R} \times \mathbb{R}^{3}$. By $(5.9)$, we see that for $(w, y, \theta, \eta)=$ $\Theta(s, v)$, the value of $y$ does not change if we change $v$. Therefore the $y$-component of $\Theta(s, v)$ is equal to $\Theta_{1}(s)$ for some smooth diffeomorphism $\Theta_{1}$ defined on a subset of $\mathbb{R}$.

Applying $\varkappa_{0}^{+}$to (5.14) and using (5.11), we get

$$
\left\{\Theta(s, v):(s, v) \in \widetilde{U},|s| \leqslant \varepsilon_{0} \tau,|v| \leqslant \nu_{1}\right\} \cap \operatorname{supp} \tilde{a}_{+}=\varnothing .
$$

However, by (5.13) we have

$$
\operatorname{diam}(\widetilde{U}) \leqslant \sqrt{C_{1}} \operatorname{diam}\left(W^{\prime \prime}\right) \leqslant \frac{1}{10} \nu_{1}
$$

and thus the condition $|v| \leqslant \nu_{1}$ in (5.15) is not needed. Therefore,

$$
\Theta_{1}^{-1}\left(\Omega_{+}\right) \cap\left[-\varepsilon_{0} \tau, \varepsilon_{0} \tau\right]=\varnothing
$$

Denote

$$
\left(w_{1}, y_{1}, \theta_{1}, \eta_{1}\right):=\Theta(0,0)=\varkappa_{0}^{+}\left(x_{1}, \xi_{1}\right) \in W^{\prime \prime},
$$

and consider the interval

$$
J:=\left[y_{1}, y_{1}+\nu|I|\right], \quad|J|=\nu|I|
$$

We have $\left|y_{0}-y_{1}\right| \leqslant C_{1} s_{0} \leqslant C_{1}^{-2} \nu_{1}|I|$. Therefore, $J \subset I$. Moreover, since $\Theta_{1}(0)=y_{1}$ and $\operatorname{diam}\left(\Theta_{1}^{-1}(J)\right) \leqslant C_{1} \nu|I| \leqslant \varepsilon_{0} \tau$, (5.16) implies that $J \cap \Omega_{+}=\varnothing$. This gives the required porosity condition on $\Omega_{+}$.

We are ready to finish the proof of Proposition 5.7. The operator $B=\mathcal{B}_{-} \mathcal{B}_{+}^{\prime}$ lies in $I_{h}^{\text {comp }}\left(\varkappa^{-} \circ\left(\varkappa^{+}\right)^{-1}\right)$. By [DZa, Lemma 4.9] we can write

$$
B=A \widetilde{\mathcal{B}}_{\chi}+\mathcal{O}\left(h^{\infty}\right)_{L^{2} \rightarrow L^{2}} \quad \text { for some } A \in \Psi_{h}^{\mathrm{comp}}\left(\mathbb{R}^{+} \times \mathbb{S}^{1}\right)
$$

where $\chi \in C_{0}^{\infty}\left(\mathbb{S}_{y}^{1} \times \mathbb{S}_{y^{\prime}}^{1}\right)$, supp $\chi \subset\left\{y \neq y^{\prime}\right\}$, and $\widetilde{\mathcal{B}}_{\chi}: L^{2}\left(\mathbb{R}^{+} \times \mathbb{S}^{1}\right) \rightarrow L^{2}\left(\mathbb{R}^{+} \times \mathbb{S}^{1}\right)$ is given by $\widetilde{\mathcal{B}}_{\chi} v(w, y)=\mathcal{B}_{\chi, w}(v(w, \cdot))(y)$, where

$$
\mathcal{B}_{\chi, w} v(y)=(2 \pi h)^{-1 / 2} \int_{\mathbb{S}^{1}}\left|\frac{y-y^{\prime}}{2}\right|^{2 i w / h} \chi\left(y, y^{\prime}\right) v\left(y^{\prime}\right) d y^{\prime}, \quad w>0 .
$$

Here $\left|y-y^{\prime}\right|$ denotes the Euclidean distance between $y, y^{\prime} \in \mathbb{S}^{1} \subset \mathbb{R}^{2}$.

Since supp $a_{ \pm} \subset\left\{\frac{1}{4} \leqslant|\xi|_{g} \leqslant 4\right\}$, we have supp $\tilde{a}_{ \pm} \subset\left\{\frac{1}{4} \leqslant w \leqslant 4\right\}$. We can write

$$
\mathrm{Op}_{h}^{L_{0}}\left(\tilde{a}_{-}\right) B \mathrm{Op}_{h}^{L_{0}}\left(\tilde{a}_{+}\right)=\mathrm{Op}_{h}^{L_{0}}\left(a_{-}^{\prime}\right) \tilde{\mathcal{B}}_{\chi} \mathrm{Op}_{h}^{L_{0}}\left(a_{+}^{\prime}\right)+\mathcal{O}\left(h^{\infty}\right)_{L^{2} \rightarrow L^{2}}
$$


where $a_{ \pm}^{\prime} \in S_{L_{0}, \varrho}^{\mathrm{comp}}\left(T^{*}\left(\mathbb{R}^{+} \times \mathbb{S}^{1}\right)\right)$ satisfy

$$
\operatorname{supp} a_{ \pm}^{\prime} \subset\left\{\frac{1}{4} \leqslant w \leqslant 4 \text { and } y \in \Omega_{ \pm}\right\}
$$

In fact, $a_{-}^{\prime}=\tilde{a}_{-} \# \sigma_{h}(A)$ and $a_{+}^{\prime}=\tilde{a}_{+}$. By [DZa, Lemma 3.3], there exist symbols $\chi_{ \pm}(y ; h)$ such that

$$
\left|\partial_{y}^{k} \chi_{ \pm}\right| \leqslant C_{k} h^{-\varrho k}, \quad \operatorname{supp}\left(1-\chi_{ \pm}\right) \cap \Omega_{ \pm}=\varnothing, \quad \operatorname{supp} \chi_{ \pm} \subset \Omega_{ \pm}\left(h^{\varrho}\right)
$$

Take also $\chi_{w}(w) \in C_{0}^{\infty}\left(\left(\frac{1}{8}, 8\right)\right)$ such that $\chi_{w}=1$ near $\left[\frac{1}{4}, 4\right]$. Then, it follows from $(5.17)$ and (5.18) that

$$
\mathrm{Op}_{h}^{L_{0}}\left(\tilde{a}_{-}\right) B \mathrm{Op}_{h}^{L_{0}}\left(\tilde{a}_{+}\right)=\mathrm{Op}_{h}^{L_{0}}\left(a_{-}^{\prime}\right) \chi_{w} \chi_{-} \widetilde{\mathcal{B}}_{\chi} \chi_{+} \mathrm{Op}_{h}^{L_{0}}\left(a_{+}^{\prime}\right)+\mathcal{O}\left(h^{\infty}\right)_{L^{2} \rightarrow L^{2}}
$$

Therefore, (5.12) follows from the estimate

$$
\left\|\chi_{w} \chi_{-} \widetilde{\mathcal{B}}_{\chi} \chi_{+}\right\|_{L^{2}\left(\mathbb{R}+\times \mathbb{S}^{1}\right) \rightarrow L^{2}\left(\mathbb{R}^{+} \times \mathbb{S}^{1}\right)} \leqslant C h^{\beta},
$$

which in turn follows from

$$
\sup _{w \in[1 / 8,8]}\left\|\mathbb{1}_{\Omega_{-}\left(h^{\varrho}\right)} \mathcal{B}_{\chi, w} \mathbb{1}_{\Omega_{+}\left(h^{\varrho}\right)}\right\|_{L^{2}\left(\mathbb{S}^{1}\right) \rightarrow L^{2}\left(\mathbb{S}^{1}\right)} \leqslant C h^{\beta}
$$

The operator $\mathcal{B}_{\chi, w}$ has the form (5.1) with

$$
\Phi\left(y, y^{\prime}\right)=2 w \log \left|y-y^{\prime}\right|-w \log 4, \quad y, y^{\prime} \in \mathbb{S}^{1}, y \neq y^{\prime},
$$

where we pass from operators on $\mathbb{S}^{1}$ to operators on $\mathbb{R}$ by taking a partition of unity for $\chi$. The mixed derivative $\partial_{y y^{\prime}}^{2} \Phi$ does not vanish as verified for instance in [BD, §4.3]. Therefore (5.19) follows from the 1-dimensional fractal uncertainty principle (Proposition 5.5), where the porosity condition for $\Omega_{ \pm}$has been verified in Lemma 5.8.

\subsection{Proof of Proposition 3.5}

We now prove Proposition 3.5. Take an arbitrary word $\mathbf{w} \in \mathcal{W}\left(2 N_{1}\right)$, and write it as a concatenation of two words in $\mathcal{W}\left(N_{1}\right)$ :

$$
\mathbf{w}=\mathbf{w}_{+} \mathbf{w}_{-}, \quad \mathbf{w}_{ \pm} \in \mathcal{W}\left(N_{1}\right)
$$

Define the operators

$$
\mathcal{A}_{+}:=A_{\mathbf{w}_{+}}\left(-N_{1}\right) \quad \text { and } \quad \mathcal{A}_{-}:=A_{\mathbf{w}_{-}} .
$$


Then,

$$
A_{\mathrm{w}}=U\left(-N_{1}\right) \mathcal{A}_{-} \mathcal{A}_{+} U\left(N_{1}\right) .
$$

We relabel the letters in the words $\mathbf{w}_{ \pm}$as follows:

$$
\mathbf{w}_{+}=w_{N_{1}}^{+} \ldots w_{1}^{+} \quad \text { and } \quad \mathbf{w}_{-}=w_{0}^{-} \ldots w_{N_{1}-1}^{-}
$$

and define the symbols $a_{ \pm}$by

$$
a_{+}=\prod_{j=1}^{N_{1}}\left(a_{w_{j}^{+}} \circ \varphi_{-j}\right) \quad \text { and } \quad a_{-}=\prod_{j=0}^{N_{1}-1}\left(a_{w_{j}^{-}} \circ \varphi_{j}\right)
$$

Recall from (3.5) that

$$
\begin{aligned}
& \mathcal{A}_{-}=A_{w_{N_{1}-1}^{-}}\left(N_{1}-1\right) A_{w_{N_{1}-2}^{-}}\left(N_{1}-2\right) \ldots A_{w_{1}^{-}}(1) A_{w_{0}^{-}}(0), \\
& \mathcal{A}_{+}=A_{w_{1}^{+}}(-1) A_{w_{2}^{+}}(-2) \ldots A_{w_{N_{1}-1}^{+}}\left(1-N_{1}\right) A_{w_{N_{1}}^{+}}\left(-N_{1}\right) .
\end{aligned}
$$

Lemma 5.9. The symbols $a_{ \pm}$and the operators $\mathcal{A}_{ \pm}$satisfy

$$
\begin{array}{ll}
a_{+} \in S_{L_{u}, \varrho}^{\mathrm{comp}}\left(T^{*} M \backslash\{0\}\right), & \mathcal{A}_{+}=\mathrm{Op}_{h}^{L_{u}}\left(a_{+}\right)+\mathcal{O}\left(h^{1-\varrho-}\right)_{L^{2} \rightarrow L^{2}}, \\
a_{-} \in S_{L_{s}, \varrho}^{\mathrm{comp}}\left(T^{*} M \backslash\{0\}\right), & \mathcal{A}_{-}=\mathrm{Op}_{h}^{L_{s}}\left(a_{-}\right)+\mathcal{O}\left(h^{1-\varrho-}\right)_{L^{2} \rightarrow L^{2}} .
\end{array}
$$

Proof. The statement for $a_{-}$and $\mathcal{A}_{-}$follows directly from Lemma 3.2. The statement for $a_{+}$and $\mathcal{A}_{+}$can be obtained similarly by reversing the flow $\varphi_{t}$ which exchanges the stable and unstable foliations.

By Lemma 5.9 and (5.20), to show Proposition 3.5 it suffices to prove the estimate

$$
\left\|\mathrm{Op}_{h}^{L_{s}}\left(a_{-}\right) \mathrm{Op}_{h}^{L_{u}}\left(a_{+}\right)\right\|_{L^{2} \rightarrow L^{2}} \leqslant C h^{\beta}
$$

The latter follows from the version of the fractal uncertainty principle in Proposition 5.7 (with $Q=I$ ), where the porosity condition is established by the following result.

Lemma 5.10. There exist $\varepsilon_{0}, \nu_{1}, K_{1}>0$, depending only on $M, \mathcal{U}_{1}$, and $\mathcal{U}_{2}$, such that the sets $\operatorname{supp} a_{ \pm}$are $\left(\varepsilon_{0}, \nu_{1}\right)$-porous up to scale $K_{1} h^{\varrho}$ along $U_{ \pm}$in the sense of Definition 5.6.

Proof. We show the porosity of $\operatorname{supp} a_{-}$. The porosity of $\operatorname{supp} a_{+}$can be proved in the same way, by reversing the direction of the flow $\varphi_{t}$.

Recall from (3.2) that $\operatorname{supp} a_{1} \cap \mathcal{U}_{1}=\operatorname{supp} a_{2} \cap \mathcal{U}_{2}=\varnothing$ where $\mathcal{U}_{1}$ and $\mathcal{U}_{2}$ are non-empty open conic subsets of $T^{*} M \backslash\{0\}$. Fix non-empty open conic subsets $\mathcal{U}_{1}^{\prime}, \mathcal{U}_{2}^{\prime} \subset T^{*} M \backslash\{0\}$ such that $\mathcal{U}_{w}^{\prime} \cap S^{*} M \Subset \mathcal{U}_{w}, w=1,2$. 
By Proposition 2.1 and since the vector field $U_{-}$is homogeneous, there is $T>1$, depending only on $M, \mathcal{U}_{1}^{\prime}$, and $\mathcal{U}_{2}^{\prime}$, such that for each $(x, \xi) \in T^{*} M \backslash\{0\}$ there exist $s_{w}=$ $s_{w}(x, \xi) \in[0, T], w=1,2$, such that

$$
e^{s_{w} U_{-}}(x, \xi) \in \mathcal{U}_{w}^{\prime}
$$

We put $K_{1}:=3 T$. Take arbitrary $(x, \xi) \in T^{*} M \backslash\{0\}$ and $\tau$ such that $K_{1} h^{\varrho} \leqslant \tau \leqslant 1$. Let $j$ be the unique integer such that $e^{j-1} \tau<T \leqslant e^{j} \tau$. Then, $1 \leqslant j \leqslant N_{1}-1$. Denote $w:=w_{j}^{-} \in\{1,2\}$, so that

$$
\left(\operatorname{supp} a_{-}\right) \cap\left(\varphi_{-j}\left(\mathcal{U}_{w}\right)\right)=\varnothing .
$$

Since $e^{j} \tau \geqslant T$, we see that there exists $s_{0}:=e^{-j} s_{w}\left(\varphi_{j}(x, \xi)\right) \in[0, \tau]$ such that

$$
q:=\varphi_{j}\left(e^{s_{0} U_{-}}(x, \xi)\right)=e^{e^{j} s_{0} U_{-}}\left(\varphi_{j}(x, \xi)\right) \in \mathcal{U}_{w}^{\prime}
$$

Here, we used the commutation relations (2.5). For $v \in \mathbb{R}^{3}$ and $s \in \mathbb{R}$ we have

$$
\varphi_{j}\left(e^{\mathcal{V}_{+} v} e^{\left(s+s_{0}\right) U_{-}}(x, \xi)\right)=e^{\mathcal{V}_{+} v^{\prime}} e^{e^{j} s U_{-}}(q),
$$

where $v^{\prime}=\left(v_{1}, v_{2}, e^{-j} v_{3}\right)$. In particular, $\left|v^{\prime}\right| \leqslant|v|$. Now, choose $\nu_{1}>0$ such that, for $w=$ 1,2 ,

$$
\max \{|v|,|s|\} \leqslant \nu_{1} \quad \Longrightarrow \quad e^{\mathcal{V}_{+} v} e^{s U_{-}}\left(\mathcal{U}_{w}^{\prime}\right) \subset \mathcal{U}_{w}
$$

and put $\varepsilon_{0}:=\nu_{1} / 3 T$. By $(5.22)$ and $(5.23)$ we have $\Sigma_{\varepsilon_{0} \tau, \nu_{1}}^{-}\left(e^{s_{0} U_{-}}(x, \xi)\right) \subset \varphi_{-j}\left(\mathcal{U}_{w}\right)$. By $(5.21)$, we then have

$$
\Sigma_{\varepsilon_{0} \tau, \nu_{1}}^{-}\left(e^{s_{0} U_{-}}(x, \xi)\right) \cap \operatorname{supp} a_{-}=\varnothing .
$$

This finishes the proof of the porosity of $\operatorname{supp} a_{-}$.

\section{Appendix A. Calculus associated with a Lagrangian foliation}

In this appendix, we establish properties of the $\Psi_{h, L, \varrho}^{\text {comp }}$ pseudodifferential calculus introduced in $\S 2.3$. We follow [DZa, §3], indicating the changes necessary. We present the calculus in the general setting of a Lagrangian foliation on an arbitrary manifold.

\section{A.1. Symbols}

We assume that $M$ is a manifold, $U \subset T^{*} M$ is an open set, and $L$ is a Lagrangian foliation, that is for each $(x, \xi) \in U, L_{(x, \xi)} \subset T_{(x, \xi)}\left(T^{*} M\right)$ is a Lagrangian subspace depending smoothly on $(x, \xi)$ and the family $\left(L_{(x, \xi)}\right)_{(x, \xi) \in U}$ is integrable. See [DZa, Definition 3.1]. 
To keep track of powers of $h$ in the remainder, we introduce a slightly more general class of symbols than the one used in $\S 2.3$. Fix two parameters

$$
0 \leqslant \varrho<1 \quad \text { and } \quad 0 \leqslant \varrho^{\prime} \leqslant \frac{1}{2} \varrho, \quad \text { with } \varrho+\varrho^{\prime}<1
$$

We say that an $h$-dependent symbol $a$ lies in the class $S_{L, \varrho, \varrho^{\prime}}^{\text {comp }}(U)$ if

(1) $a(x, \xi ; h)$ is smooth in $(x, \xi) \in U$, defined for $0<h \leqslant 1$, and supported in an $h$ independent compact subset of $U$;

(2) a satisfies the derivative bounds

$$
\sup _{x, \xi}\left|Y_{1} \ldots Y_{m} Z_{1} \ldots Z_{k} a(x, \xi ; h)\right| \leqslant C h^{-\varrho k-\varrho^{\prime} m}, \quad 0<h \leqslant 1
$$

for all vector fields $Y_{1}, \ldots, Y_{m}, Z_{1}, \ldots, Z_{k}$ on $U$ such that $Y_{1}, \ldots, Y_{m}$ are tangent to $L$. Here the constant $C$ depends on $Y_{1}, \ldots, Y_{m}, Z_{1}, \ldots, Z_{k}$, but does not depend on $h$.

For $\varrho^{\prime}=0$ we obtain the class used in [DZa, $\left.\S 3\right]$. Moreover, the class $S_{L, \varrho}^{\mathrm{comp}}\left(T^{*} M \backslash\{0\}\right)$ introduced in $\S 2.3$ is given by

$$
S_{L, \varrho}^{\mathrm{comp}}\left(T^{*} M \backslash\{0\}\right)=\bigcap_{\varepsilon>0} S_{L, \varrho+\varepsilon, \varepsilon}^{\mathrm{comp}}\left(T^{*} M \backslash\{0\}\right)
$$

In the arguments below (for instance, in (A.8), (A.11), and (A.19)) we implicitly use the following version of Borel's theorem (see [Zw1, Theorem 4.15] for the standard version whose proof applies here). Let $a_{j} \in S_{L, \varrho, \varrho^{\prime}}^{\text {comp }}(U)$ be a sequence of symbols with supports contained in a compact subset of $U$ independent of $h$ and $j$. Take an increasing sequence of real numbers $m_{j} \geqslant 0, m_{j} \rightarrow \infty$. Then, there exists a symbol $a \in S_{L, \varrho, \varrho^{\prime}}^{\text {comp }}(U)$ which is an asymptotic sum of $h^{m_{j}} a_{j}$ in the following sense:

$$
a-\sum_{j=0}^{J-1} h^{m_{j}} a_{j} \in h^{m_{J}} S_{L, \varrho, \varrho^{\prime}}^{\mathrm{comp}}(U) \quad \text { for all } J,
$$

and moreover supp $a \subset \bigcup_{j} \operatorname{supp} a_{j}$. Here, supp $a$ denotes the support of $a$ in the $(x, \xi)$ variables, which is an $h$-dependent family of compact subsets of $U$.

We have the following bound for the product of many symbols in $S_{L, \varrho, \varrho^{\prime}}^{\mathrm{comp}}(U)$.

LEMmA A.1. Let $C$ be an arbitrary fixed constant and assume that $a_{1}, \ldots, a_{N} \in$ $S_{L, \varrho, \varrho^{\prime}}^{\text {comp }}(U), 1 \leqslant N \leqslant C \log (1 / h)$ are such that $\sup \left|a_{j}\right| \leqslant 1$ and each $S_{L, \varrho, \varrho^{\prime}}^{\text {comp }}(U)$ seminorm of $a_{j}$ is bounded uniformly in $j$. Then, for all small $\varepsilon>0$, the product $a_{1} \ldots a_{N}$ lies in $S_{L, \varrho+\varepsilon, \varrho^{\prime}+\varepsilon}^{\mathrm{comp}}(U)$. 
Proof. We see immediately that $\sup \left|a_{1} \ldots a_{N}\right| \leqslant 1$ and $\operatorname{supp}\left(a_{1} \ldots a_{N}\right) \subset \operatorname{supp} a_{1}$ lies in an $h$-independent compact subset of $U$. It remains to verify that, for all vector fields $Y_{1}, \ldots, Y_{m}, Z_{1}, \ldots, Z_{k}$ on $U$ such that $Y_{1}, \ldots, Y_{m}$ are tangent to $L$ and each $\varepsilon>0$,

$$
\sup _{x, \xi}\left|Y_{1} \ldots Y_{m} Z_{1} \ldots Z_{k}\left(a_{1} \ldots a_{N}\right)\right|=\mathcal{O}\left(h^{-\varrho k-\varrho^{\prime} m-\varepsilon}\right) .
$$

By the Leibniz rule, $Y_{1} \ldots Y_{m} Z_{1} \ldots Z_{k}\left(a_{1} \ldots a_{N}\right)$ is a sum of $N^{m+k}=\mathcal{O}\left(h^{-\varepsilon}\right)$ terms. Each of these summands is a product of $N$ terms, of which at least $N-m-k$ have the form $a_{j}$ for some $j$, and the rest are obtained by differentiating $a_{j}$. Since the $S_{L, \varrho, \varrho^{\prime}}^{\text {comp }}(U)$ seminorms of $a_{j}$ are bounded uniformly in $j$, each summand is $\mathcal{O}\left(h^{-\varrho k-\varrho^{\prime} m}\right)$, giving (A.3).

\section{A.2. Model calculus}

In $\S \S \mathrm{A} .2-\mathrm{A} .4$ we review the construction of the calculus in [DZa, $\S \S 3.2,3.3]$, explaining how to modify it to quantize symbols in $S_{L, \varrho, \varrho^{\prime}}^{\text {comp }}(U)$.

Following [DZa, $§ 3.2]$, we first consider the model case when $M=\mathbb{R}^{n}, U=T^{*} \mathbb{R}^{n}$, and $L=L_{0}$ is the vertical foliation:

$$
L_{0}=\operatorname{span}\left(\partial_{\eta_{1}}, \ldots, \partial_{\eta_{n}}\right) \text {, }
$$

where $(y, \eta)$ are the standard coordinates on $T^{*} \mathbb{R}^{n}$. Symbols in $S_{L_{0}, \varrho, \varrho^{\prime}}^{\text {comp }}\left(T^{*} \mathbb{R}^{n}\right)$ satisfy the derivative bounds

$$
\sup _{y, \eta}\left|\partial_{y}^{\alpha} \partial_{\eta}^{\beta} a(y, \eta ; h)\right| \leqslant C_{\alpha \beta} h^{-\varrho|\alpha|-\varrho^{\prime}|\beta|} .
$$

For these symbols we use the standard quantization

$$
\mathrm{Op}_{h}(a) f(y)=(2 \pi h)^{-n} \int_{\mathbb{R}^{2 n}} e^{(i / h)\left(y-y^{\prime}\right) \cdot \eta} a(y, \eta) f\left(y^{\prime}\right) d y^{\prime} d \eta
$$

Other quantizations such as the Weyl quantization are likely to produce the same class of operators, however the standard quantization is convenient for proving invariance under conjugation by Fourier integral operators; see [DZa, Lemma 3.10].

The standard quantization has the following properties:

(1) for $a \in S_{L_{0}, \varrho, \varrho^{\prime}}^{\text {comp }}\left(T^{*} \mathbb{R}^{n}\right)$ the operator $\operatorname{Op}_{h}(a): L^{2}\left(\mathbb{R}^{n}\right) \rightarrow L^{2}\left(\mathbb{R}^{n}\right)$ is bounded uniformly in $h$;

(2) for $a, b \in S_{L_{0}, \varrho, \varrho^{\prime}}^{\text {comp }}\left(T^{*} \mathbb{R}^{n}\right)$ we have, for some $a \# b \in S_{L_{0}, \varrho, \varrho^{\prime}}^{\text {comp }}\left(T^{*} \mathbb{R}^{n}\right)$,

$$
\begin{aligned}
\mathrm{Op}_{h}(a) \mathrm{Op}_{h}(b) & =\mathrm{Op}_{h}(a \# b)+\mathcal{O}\left(h^{\infty}\right)_{L^{2} \rightarrow L^{2}}, \\
a \# b & =a b+\mathcal{O}\left(h^{1-\varrho-\varrho^{\prime}}\right)_{L_{L_{0}, \varrho, \varrho^{\prime}}}\left(T^{*} \mathbb{R}^{n}\right) \\
\operatorname{supp}(a \# b) & \subset \operatorname{supp} a \cap \operatorname{supp} b
\end{aligned}
$$


(3) for $a \in S_{L_{0}, \varrho, \varrho^{\prime}}^{\text {comp }}\left(T^{*} \mathbb{R}^{n}\right)$ we have, for some $a^{*} \in S_{L_{0}, \varrho, \varrho^{\prime}}^{\text {comp }}\left(T^{*} \mathbb{R}^{n}\right)$,

$$
\begin{aligned}
\mathrm{Op}_{h}(a)^{*} & =\operatorname{Op}_{h}\left(a^{*}\right)+\mathcal{O}\left(h^{\infty}\right)_{L^{2} \rightarrow L^{2}} \\
a^{*} & =\bar{a}+\mathcal{O}\left(h^{1-\varrho-\varrho^{\prime}}\right)_{S_{0}, \varrho, \varrho^{\prime}}{ }^{\mathrm{comp}}\left(T^{*} \mathbb{R}^{n}\right) \\
\operatorname{supp} a^{*} & \subset \operatorname{supp} a
\end{aligned}
$$

(4) if one of the symbols $a$ and $b$ lies in $S_{L_{0}, \varrho, \varrho^{\prime}}^{\text {comp }}\left(T^{*} \mathbb{R}^{n}\right)$, and the other one has all derivatives bounded uniformly in $h$ (it does not have to be compactly supported), then (A.6) and (A.8) hold, and

$$
a \# b=a b+\mathcal{O}\left(h^{1-\varrho}\right)_{S_{L_{0}, \varrho, \varrho^{\prime}}^{\mathrm{comp}}\left(T^{*} \mathbb{R}^{n}\right)}
$$

(5) if $a=a_{0}+h a_{1}$, where $a_{0}$ and $a_{1}$ have all derivatives bounded uniformly in $h$, $b=b_{0}+h^{1-\varrho} b_{1}$, where $b_{0}, b_{1} \in S_{L_{0}, \varrho, \varrho^{\prime}}^{\text {comp }}\left(T^{*} \mathbb{R}^{n}\right)$, and $\partial_{\eta} a_{0}=0$ near supp $b_{0} \cup \operatorname{supp} b_{1}$, then

$$
a \# b-b \# a=-i h\left\{a_{0}, b_{0}\right\}+\mathcal{O}\left(h^{2-\varrho-\varrho^{\prime}}\right)_{S_{L_{0}, \varrho, \varrho^{\prime}}^{\mathrm{comp}}\left(T^{*} \mathbb{R}^{n}\right)} .
$$

The proofs are similar to those of [DZa, Lemmas 3.7, 3.8]. More precisely, we use the unitary rescaling operator

$$
T_{\varrho, \varrho^{\prime}}: L^{2}\left(\mathbb{R}^{n}\right) \longrightarrow L^{2}\left(\mathbb{R}^{n}\right), \quad T_{\varrho, \varrho^{\prime}} u(y)=h^{\left(\varrho-\varrho^{\prime}\right) n / 4} u\left(h^{\left(\varrho-\varrho^{\prime}\right) / 2} y\right),
$$

to conjugate $\mathrm{Op}_{h}(a)$ as follows:

$$
T_{\varrho, \varrho^{\prime}} \mathrm{Op}_{h}(a) T_{\varrho, \varrho^{\prime}}^{-1}=\mathrm{Op}_{h}\left(a_{\varrho, \varrho^{\prime}}\right), \quad a_{\varrho, \varrho^{\prime}}(y, \eta ; h):=a\left(h^{\left(\varrho-\varrho^{\prime}\right) / 2} y, h^{\left(\varrho^{\prime}-\varrho\right) / 2} \eta ; h\right) .
$$

If $a$ satisfies (A.4), then the rescaled symbol $a_{\varrho, \varrho^{\prime}}$ satisfies

$$
\sup _{y, \eta}\left|\partial_{y}^{\alpha} \partial_{\eta}^{\beta} a_{\varrho, \varrho^{\prime}}(y, \eta ; h)\right| \leqslant C_{\alpha \beta} h^{-\left(\varrho+\varrho^{\prime}\right)(|\alpha|+|\beta|) / 2}
$$

that is $a_{\varrho, \varrho^{\prime}} \in S_{\left(\varrho+\varrho^{\prime}\right) / 2}$, where the classes $S_{\delta}, 0 \leqslant \delta \leqslant \frac{1}{2}$, are defined in [Zw1, formula (4.4.5)]. Then the statements (1)-(3) above follow from the standard properties of the $S_{\delta}$ calculus; see [Zw1, Theorems 4.23 (ii), 4.14, and 4.17]. The statements (4) and (5) follow by an examination of the terms in the asymptotic expansion for $a \# b$.

The model calculus satisfies the following version of sharp Gårding inequality.

Lemma A.2. Assume that $a \in S_{L_{0}, \varrho, \varrho^{\prime}}^{\text {comp }}\left(T^{*} \mathbb{R}^{n}\right)$ satisfies $\operatorname{Re} a \geqslant 0$ everywhere. Then, there exists a constant $C$ depending on a such that, for all $h$ and all $u \in L^{2}\left(\mathbb{R}^{n}\right)$,

$$
\operatorname{Re}\left\langle\mathrm{Op}_{h}(a) u, u\right\rangle_{L^{2}} \geqslant-C h^{1-\varrho-\varrho^{\prime}}\|u\|_{L^{2}}^{2}
$$


Proof. We take the following rescaled versions of $u, a$, and $h$ :

$$
\tilde{u}(y):=h^{\varrho n / 2} u\left(h^{\varrho} y\right), \quad \tilde{a}(y, \eta ; h):=a\left(h^{\varrho} y, h^{\varrho^{\prime}} \eta ; h\right), \quad \text { and } \quad \tilde{h}:=h^{1-\varrho-\varrho^{\prime}} .
$$

Note that $\|\tilde{u}\|_{L^{2}}=\|u\|_{L^{2}}$. We have

$$
\operatorname{Re}\left\langle\mathrm{Op}_{h}(a) u, u\right\rangle_{L^{2}}=\operatorname{Re}\left\langle\mathrm{Op}_{\tilde{h}}(\tilde{a}) \tilde{u}, \tilde{u}\right\rangle_{L^{2}}
$$

Now (A.4) implies that all derivatives of $\tilde{a}$ are bounded uniformly in $h$. It remains to apply the standard sharp Gårding inequality [Zw1, Theorem 4.32].

\section{A.3. Fourier integral operators}

To pass from the model case to the general case, we study the conjugation of operators in the model calculus by Fourier integral operators. We briefly review the notation for Fourier integral operators, referring the reader to [DZa, §2.2] and the references there for details.

- Let $M_{1}$ and $M_{2}$ be manifolds of the same dimension. An exact symplectomorphism is a diffeomorphism $\varkappa$ : $U_{2} \rightarrow U_{1}$, where $U_{j} \subset T^{*} M_{j}$ are open sets, such that $\varkappa^{*}(\xi d x)-\eta d y$ is an exact 1-form. Here $\xi d x$ and $\eta d y$ are the canonical 1-forms on $T^{*} M_{1}$ and $T^{*} M_{2}$, respectively. We fix an antiderivative for $\varkappa^{*}(\xi d x)-\eta d y$.

- For an exact symplectomorphism $\varkappa$ (with fixed antiderivative), denote by $I_{h}^{\text {comp }}(\varkappa)$ the class of compactly supported $\left(^{1}\right)$ and compactly microlocalized semiclassical Fourier integral operators associated with $\varkappa$. These operators are bounded $L^{2}\left(M_{2}\right) \rightarrow L^{2}\left(M_{1}\right)$ uniformly in $h$.

- Let $\varkappa: U_{2} \rightarrow U_{1}$ be an exact symplectomorphism and $\varkappa^{-1}$ denote its inverse. For any $B \in I_{h}^{\text {comp }}(\varkappa)$ and $B^{\prime} \in I_{h}^{\text {comp }}\left(\varkappa^{-1}\right)$, the operators $B B^{\prime}$ and $B^{\prime} B$ are pseudodifferential in the class $\Psi_{h}^{\text {comp }}$ and [DZa, formula $\left.(2.12)\right]$

$$
\sigma_{h}\left(B^{\prime} B\right)=\sigma_{h}\left(B B^{\prime}\right) \circ \varkappa
$$

If $V_{1} \subset U_{1}$ and $V_{2} \subset U_{2}$ are compact sets such that $\varkappa\left(V_{2}\right)=V_{1}$, then we say that $B$ and $B^{\prime}$ quantize $\varkappa$ near $V_{1} \times V_{2}$ if

$$
\begin{array}{ll}
B B^{\prime}=I+\mathcal{O}\left(h^{\infty}\right) & \text { microlocally near } V_{1}, \\
B^{\prime} B=I+\mathcal{O}\left(h^{\infty}\right) & \text { microlocally near } V_{2} .
\end{array}
$$

$\left({ }^{1}\right)$ An operator is called compactly supported if its Schwartz kernel is compactly supported. 
The quantization studied in $\S$ A.2 is invariant under conjugation by Fourier integral operators whose underlying symplectomorphisms preserve $L_{0}$.

Lemma A.3. Assume that $\varkappa: U_{2} \rightarrow U_{1}, U_{j} \subset T^{*} \mathbb{R}^{n}$, is an exact symplectomorphism such that $\varkappa_{*}\left(L_{0}\right)=L_{0}$, and take $B \in I_{h}^{\text {comp }}(\varkappa)$ and $B^{\prime} \in I_{h}^{\text {comp }}\left(\varkappa^{-1}\right)$. Then, for each $a \in$ $S_{L_{0}, \varrho, \varrho^{\prime}}^{\text {comp }}\left(T^{*} \mathbb{R}^{n}\right)$, there exists $b \in S_{L_{0}, \varrho, \varrho^{\prime}}^{\text {comp }}\left(T^{*} \mathbb{R}^{n}\right)$ such that

$$
\begin{aligned}
B^{\prime} \mathrm{Op}_{h}(a) B & =\mathrm{Op}_{h}(b)+\mathcal{O}\left(h^{\infty}\right)_{L^{2} \rightarrow L^{2}}, \\
b & =(a \circ \varkappa) \sigma_{h}\left(B^{\prime} B\right)+\mathcal{O}\left(h^{1-\varrho}\right)_{S_{L_{0}, \varrho, \varrho^{\prime}}^{\mathrm{comp}}\left(T^{*} \mathbb{R}^{n}\right)}, \\
\operatorname{supp} b & \subset \varkappa^{-1}(\operatorname{supp} a) .
\end{aligned}
$$

Proof. We argue exactly as in the proofs of [DZa, Lemmas 3.9 and 3.10]. The stationary phase asymptotic at the end of the proof of [DZa, Lemma 3.9] produces a remainder $\mathcal{O}\left(h^{1-2 \varrho^{\prime}}\right)$. The multiplication formula (A.12) applied to the expression $A^{\prime} \mathrm{Op}_{h}(\tilde{a}) A$ in the last paragraph of the proof of [DZa, Lemma 3.10] gives a remainder $\mathcal{O}\left(h^{1-\varrho}\right)$.

\section{A.4. General calculus}

We now construct a quantization $\operatorname{Op}_{h}^{L}(a)$ of a symbol $a \in S_{L, \varrho, \varrho^{\prime}}^{\text {comp }}(U)$ for a general Lagrangian foliation $L$ on $U \subset T^{*} M$. This is done similarly to [DZa, §3.3] by summing operators in the model calculus conjugated by appropriately chosen Fourier integral operators.

We say that $\left(U^{\prime}, \varkappa, B, B^{\prime}\right)$ is a chart for $L$ if

- $U^{\prime} \subset U$ is an open set and $\varkappa: U^{\prime} \rightarrow T^{*} \mathbb{R}^{n}$ is an exact symplectomorphism onto its image which maps $L$ to $L_{0}$;

- $B \in I_{h}^{\mathrm{comp}}(\varkappa)$ and $B^{\prime} \in I_{h}^{\mathrm{comp}}\left(\varkappa^{-1}\right)$.

For each $\left(x_{0}, \xi_{0}\right) \in U$ there exists a chart $\left(U^{\prime}, \varkappa, B, B^{\prime}\right)$ such that $\sigma_{h}\left(B^{\prime} B\right)\left(x_{0}, \xi_{0}\right) \neq 0$ (in fact, we may take $B^{\prime}=B^{*}$ ). Here, the existence of $\varkappa$ follows from [DZa, Lemma 3.6] and the existence of $B$ and $B^{\prime}$ is discussed in the paragraph following [DZa, (2.12)].

Following [DZa, formula (3.11)], we put, for $a \in S_{L, \varrho, \varrho^{\prime}}^{\mathrm{comp}}(U)$,

$$
\mathrm{Op}_{h}^{L}(a):=\sum_{\ell} B_{\ell}^{\prime} \mathrm{Op}_{h}\left(a_{\ell}\right) B_{\ell}, \quad a_{\ell}=\left(\chi_{\ell} a\right) \circ \varkappa_{\ell}^{-1} \in S_{L_{0}, \varrho, \varrho^{\prime}}^{\mathrm{comp}}\left(T^{*} \mathbb{R}^{n}\right)
$$

where $\left(U_{\ell}, \varkappa_{\ell}, B_{\ell}, B_{\ell}^{\prime}\right)$ is a collection of charts for $L$ such that $U_{\ell} \subset U$ form a locally finite cover of $U$, the symbols $\sigma_{h}\left(B_{\ell}^{\prime} B_{\ell}\right) \in C_{0}^{\infty}\left(U_{\ell}\right)$ form a partition of unity on $U, \chi_{\ell} \in$ $C_{0}^{\infty}\left(U_{\ell}\right)$ are equal to 1 near $\operatorname{supp} \sigma_{h}\left(B_{\ell}^{\prime} B_{\ell}\right)$, and $\mathrm{Op}_{h}$ is defined in (A.5). The quantization 
procedure $\mathrm{Op}_{h}^{L}$ depends on the choice of charts, however properties (3) and (4) below show that the resulting class of operators is invariant.

To simplify the proof of Lemma A.4 below, we additionally assume that $B_{\ell}^{\prime}=B_{\ell}^{*}$. This can be arranged as follows: note that for any choice of $\varkappa$ and $B \in I_{h}^{\text {comp }}(\varkappa)$, we have $B^{*} \in I_{h}^{\text {comp }}\left(\varkappa^{-1}\right)$ and $\sigma_{h}\left(B^{*} B\right) \geqslant 0$ (since $B^{*} B$ is a pseudodifferential operator which is non-negative on $\left.L^{2}\right)$. Choose a collection of charts $\left(U_{\ell}, \varkappa_{\ell}, \widetilde{B}_{\ell}, \widetilde{B}_{\ell}^{*}\right)$ for $L$ such that $b:=$ $\sum_{\ell} \sigma_{h}\left(\widetilde{B}_{\ell}^{*} \widetilde{B}_{\ell}\right)>0$ on $U$. Putting $B_{\ell}:=\widetilde{B}_{\ell} Y_{\ell}$, where $Y_{\ell} \in \Psi_{h}^{\text {comp }}(M)$ satisfy $\sigma_{h}\left(Y_{\ell}\right)=b^{-1 / 2}$ near $\mathrm{WF}_{h}\left(\widetilde{B}_{\ell}^{*} \widetilde{B}_{\ell}\right)$, we obtain $\sum_{\ell} \sigma_{h}\left(B_{\ell}^{*} B_{\ell}\right)=1$ on $U$.

For a compactly supported operator $A: L^{2}(M) \rightarrow L^{2}(M)$, we say that $A \in \Psi_{h, L, \varrho, \varrho^{\prime}}^{\text {comp }}(U)$ if $A=\mathrm{Op}_{h}^{L}(a)+\mathcal{O}\left(h^{\infty}\right)_{L^{2} \rightarrow L^{2}}$ for some $a \in S_{L, \varrho, \varrho^{\prime}}^{\text {comp }}(U)$. The quantization procedure Op ${ }_{h}^{L}$ has the following properties which are consequences of the results of $\S \mathrm{A} .2$ and $\S \mathrm{A} .3$ (see [DZa, Lemmas 3.12 and 3.14]).

(1) For each $a \in S_{L, \varrho, \varrho^{\prime}}^{\text {comp }}(U)$ the operator $\mathrm{Op}_{h}^{L}(a): L^{2}(M) \rightarrow L^{2}(M)$ is compactly supported and bounded uniformly in $h$.

(2) If $a \in C_{0}^{\infty}(U)$ is $h$-independent, then $\operatorname{Op}_{h}^{L}(a) \in \Psi_{h}^{\text {comp }}(M)$ and $\sigma_{h}\left(\mathrm{Op}_{h}^{L}(a)\right)=a$.

(3) For every $a \in S_{L, \varrho, \varrho^{\prime}}^{\text {comp }}(U)$ and every chart $\left(U^{\prime}, \varkappa, B, B^{\prime}\right)$ for $L$ there exists $b \in$ $S_{L_{0}, \varrho, \varrho^{\prime}}^{\text {comp }}\left(T^{*} \mathbb{R}^{n}\right)$ such that

$$
\begin{aligned}
B \mathrm{Op}_{h}^{L}(a) B^{\prime} & =\mathrm{Op}_{h}(b)+\mathcal{O}\left(h^{\infty}\right)_{L^{2} \rightarrow L^{2}}, \\
b & =\left(a \circ \varkappa^{-1}\right) \sigma_{h}\left(B B^{\prime}\right)+\mathcal{O}\left(h^{1-\varrho}\right)_{S_{L_{0}, \varrho, \varrho^{\prime}}}\left(T^{*} \mathbb{R}^{n}\right) \\
\operatorname{supp} b & \subset \varkappa(\operatorname{supp} a) .
\end{aligned}
$$

(4) For every $b \in S_{L_{0}, \varrho, \varrho^{\prime}}^{\text {comp }}\left(T^{*} \mathbb{R}^{n}\right)$ and every chart $\left(U^{\prime}, \varkappa, B, B^{\prime}\right)$ for $L$ there exists $a \in S_{L, \varrho, \varrho^{\prime}}^{\mathrm{comp}}(U)$ such that

$$
\begin{aligned}
B^{\prime} \mathrm{Op}_{h}(b) B & =\mathrm{Op}_{h}^{L}(a)+\mathcal{O}\left(h^{\infty}\right)_{L^{2} \rightarrow L^{2}}, \\
a & =(b \circ \varkappa) \sigma_{h}\left(B^{\prime} B\right)+\mathcal{O}\left(h^{1-\varrho}\right)_{S_{L_{0}, \varrho, \varrho^{\prime}}}\left(T^{*} \mathbb{R}^{n}\right), \\
\operatorname{supp} a & \subset \varkappa^{-1}(\operatorname{supp} b) .
\end{aligned}
$$

(5) Assume that $M_{1}$ and $M_{2}$ are manifolds of the same dimension, $U_{j} \subset T^{*} M_{j}$ are open sets, $L_{j}$ are Lagrangian foliations on $U_{j}, U_{j}^{\prime} \subset U_{j}$ are open sets, $\varkappa: U_{2}^{\prime} \rightarrow U_{1}^{\prime}$ is an exact symplectomorphism mapping $L_{2}$ to $L_{1}$, and $B \in I_{h}^{\text {comp }}(\varkappa)$ and $B^{\prime} \in I_{h}^{\text {comp }}\left(\varkappa^{-1}\right)$. Then, for each $a_{1} \in S_{L_{1}, \varrho, \varrho^{\prime}}^{\text {comp }}\left(U_{1}\right)$, there exists $a_{2} \in S_{L_{2}, \varrho, \varrho^{\prime}}^{\text {comp }}\left(U_{2}\right)$ such that

$$
\begin{aligned}
B^{\prime} \mathrm{Op}_{h}^{L_{1}}\left(a_{1}\right) B & =\mathrm{Op}_{h}^{L_{2}}\left(a_{2}\right)+\mathcal{O}\left(h^{\infty}\right)_{L^{2} \rightarrow L^{2}}, \\
a_{2} & =\left(a_{1} \circ \varkappa\right) \sigma_{h}\left(B^{\prime} B\right)+\mathcal{O}\left(h^{1-\varrho}\right)_{S_{L_{0}, \varrho, \varrho^{\prime}}}^{\text {comp }}\left(U_{2}\right), \\
\operatorname{supp} a_{2} & \subset \varkappa^{-1}\left(\operatorname{supp} a_{1}\right) .
\end{aligned}
$$


(6) For each $a, b \in S_{L, \varrho, \varrho^{\prime}}^{\mathrm{comp}}(U)$ there exists $a \#_{L} b \in S_{L, \varrho, \varrho^{\prime}}^{\mathrm{comp}}(U)$ such that

$$
\begin{aligned}
\mathrm{Op}_{h}^{L}(a) \mathrm{Op}_{h}^{L}(b) & =\mathrm{Op}_{h}^{L}\left(a \#_{L} b\right)+\mathcal{O}\left(h^{\infty}\right)_{L^{2} \rightarrow L^{2}}, \\
a \#_{L} b & =a b+\mathcal{O}\left(h^{1-\varrho-\varrho^{\prime}}\right)_{S_{L, \varrho, \varrho^{\prime}}^{\mathrm{comp}}(U)}, \\
\operatorname{supp}\left(a \#{ }_{L} b\right) & \subset \operatorname{supp} a \cap \operatorname{supp} b .
\end{aligned}
$$

(7) For each $a \in S_{L, \varrho, \varrho^{\prime}}^{\mathrm{comp}}(U)$ there exists $a_{L}^{*} \in S_{L, \varrho, \varrho^{\prime}}^{\mathrm{comp}}(U)$ such that

$$
\begin{aligned}
\mathrm{Op}_{h}^{L}(a)^{*} & =\mathrm{Op}_{h}^{L}\left(a_{L}^{*}\right)+\mathcal{O}\left(h^{\infty}\right)_{L^{2} \rightarrow L^{2}}, \\
a_{L}^{*} & =\bar{a}+\mathcal{O}\left(h^{1-\varrho-\varrho^{\prime}}\right)_{S_{L, \varrho, \varrho^{\prime}}^{\mathrm{comp}}(U)}, \\
\operatorname{supp} a_{L}^{*} & \subset \operatorname{supp} a
\end{aligned}
$$

The following version of sharp Gårding inequality follows immediately from Lemma A.2 and the fact that $B_{\ell}^{\prime}=B_{\ell}^{*}$.

Lemma A.4. Assume that $M$ is compact, $a \in S_{L, \varrho, \varrho^{\prime}}^{\mathrm{comp}}(U)$, and $\operatorname{Re} a \geqslant 0$. Then, there exists a constant $C$ depending on some $S_{L, \varrho, \varrho^{\prime}}^{\mathrm{comp}}$ seminorm of a such that

$$
\operatorname{Re}\left\langle\operatorname{Op}_{h}^{L}(a) u, u\right\rangle_{L^{2}} \geqslant-C h^{1-\varrho-\varrho^{\prime}}\|u\|_{L^{2}}^{2} \quad \text { for all } u \in L^{2}(M) .
$$

Lemma A.4 implies a more precise bound on the operator norm of $\operatorname{Op}_{h}^{L}(a)$.

Lemma A.5. Assume that $M$ is compact, $a \in S_{L, \varrho, \varrho^{\prime}}^{\mathrm{comp}}(U)$, and $\sup |a| \leqslant 1$. Then, there exists a constant $C$ depending on some $S_{L, \varrho, \varrho^{\prime}}^{\mathrm{comp}}$ seminorm of a such that

$$
\left\|\mathrm{Op}_{h}^{L}(a)\right\|_{L^{2} \rightarrow L^{2}} \leqslant 1+C h^{1-\varrho-\varrho^{\prime}} .
$$

Proof. Fix an $h$-independent $\chi \in C_{0}^{\infty}(U ;[0,1])$ such that $\chi=1$ near $\operatorname{supp} a$. Define $b:=\chi^{2}-|a|^{2}$. Then, $b \in S_{L, \varrho, \varrho^{\prime}}^{\mathrm{comp}}(U)$ and $b \geqslant 0$. Applying Lemma A.4 to $b$, we get, for all $u \in L^{2}(M)$,

$$
\begin{aligned}
\left\|\mathrm{Op}_{h}^{L}(\chi) u\right\|_{L^{2}}^{2}-\left\|\mathrm{Op}_{h}^{L}(a) u\right\|_{L^{2}}^{2} & \geqslant \operatorname{Re}\left\langle\mathrm{Op}_{h}^{L}(b) u, u\right\rangle_{L^{2}}-C h^{1-\varrho-\varrho^{\prime}}\|u\|_{L^{2}}^{2} \\
& \geqslant-C h^{1-\varrho-\varrho^{\prime}}\|u\|_{L^{2}}^{2} .
\end{aligned}
$$

Estimating the norm of $\mathrm{Op}_{h}^{L}(\chi)$ by $(2.10)$, we get

$$
\left\|\mathrm{Op}_{h}^{L}(a) u\right\|_{L^{2}}^{2} \leqslant\left\|\mathrm{Op}_{h}^{L}(\chi) u\right\|_{L^{2}}^{2}+C h^{1-\varrho-\varrho^{\prime}}\|u\|_{L^{2}}^{2} \leqslant\|u\|_{L^{2}}^{2}+C h^{1-\varrho-\varrho^{\prime}}\|u\|_{L^{2}}^{2},
$$

finishing the proof.

Using Lemma A.5, we get the following operator version of Lemma A.1. 
Lemma A.6. Let $a_{1}, \ldots, a_{N} \in S_{L, \varrho, \varrho^{\prime}}^{\mathrm{comp}}(U)$ be as in Lemma A.1 and let $A_{1}, \ldots, A_{N}$ be operators on $L^{2}(M)$ such that $A_{j}=\mathrm{Op}_{h}^{L}\left(a_{j}\right)+\mathcal{O}\left(h^{1-\varrho-\varrho^{\prime}-}\right)_{L^{2} \rightarrow L^{2}}$, where the constants in $\mathcal{O}(\cdot)$ are independent of $j$. Then,

$$
A_{1} \ldots A_{N}=\mathrm{Op}_{h}^{L}\left(a_{1} \ldots a_{N}\right)+\mathcal{O}\left(h^{1-\varrho-\varrho^{\prime}-}\right)_{L^{2} \rightarrow L^{2}} .
$$

Proof. We have

$$
A_{1} \ldots A_{N}-\mathrm{Op}_{h}^{L}\left(a_{1} \ldots a_{N}\right)=\sum_{j=1}^{N} B_{j} A_{j+1} \ldots A_{N}
$$

and

$$
B_{j}:= \begin{cases}A_{1}-\mathrm{Op}_{h}\left(a_{1}\right), & \text { if } j=1, \\ \mathrm{Op}_{h}^{L}\left(a_{1} \ldots a_{j-1}\right) A_{j}-\mathrm{Op}_{h}^{L}\left(a_{1} \ldots a_{j}\right), & \text { if } 2 \leqslant j \leqslant N .\end{cases}
$$

Here, $\operatorname{Op}_{h}^{L}\left(a_{1} \ldots a_{j-1}\right)$ is well defined since, by Lemma A.1, $a_{1} \ldots a_{j-1} \in S_{L, \varrho+\varepsilon, \varrho^{\prime}+\varepsilon}^{\mathrm{comp}}(U)$ uniformly in $j$ for any small $\varepsilon>0$.

Since $\sup \left|a_{j}\right| \leqslant 1$, by Lemma A.5 we have, for some $C$ independent of $j$,

$$
\left\|A_{j}\right\|_{L^{2} \rightarrow L^{2}} \leqslant 1+C h^{1-\varrho-\varrho^{\prime}} .
$$

Since $N=\mathcal{O}(\log (1 / h))$, we have, uniformly in $j$,

$$
\left\|A_{j+1} \ldots A_{N}\right\|_{L^{2} \rightarrow L^{2}} \leqslant C .
$$

Therefore, it suffices to show that we have, uniformly in $j$,

$$
\left\|B_{j}\right\|_{L^{2} \rightarrow L^{2}}=\mathcal{O}\left(h^{1-\varrho-\varrho^{\prime}-}\right)_{L^{2} \rightarrow L^{2}} .
$$

For $j=1$ this is immediate, so we assume $2 \leqslant j \leqslant N$. We may replace $A_{j}$ by $\operatorname{Op}_{h}^{L}\left(a_{j}\right)$ in the definition of $B_{j}$. Then (A.27) follows from the product formula (A.23) on the space $S_{L, \varrho+\varepsilon, \varrho^{\prime}+\varepsilon}^{\text {comp }}$.

\section{A.5. Egorov's theorem}

We finally prove two versions of Egorov's theorem for the $\Psi_{h, L, \varrho, \varrho^{\prime}}^{\text {comp }}(U)$ calculus. In this subsection we assume that $M$ is a compact manifold, $U \subset T^{*} M$ is open, $L$ is a Lagrangian foliation on $U$, and $P \in \Psi_{h}^{\text {comp }}(M)$ is self-adjoint with principal symbol $p=$ $\sigma_{h}(P) \in C_{0}^{\infty}\left(T^{*} M ; \mathbb{R}\right)$. We moreover assume that

$$
L_{(x, \xi)} \subset \operatorname{ker} d p(x, \xi) \quad \text { for all }(x, \xi) \in U ;
$$

this is equivalent to the Hamiltonian vector field $H_{p}$ lying inside $L$. The operator

$$
e^{-i t P / h}: L^{2}(M) \longrightarrow L^{2}(M)
$$

is unitary.

We start with the following fixed-time statement similar to [DZa, Lemma 3.17]. 
LEMmA A.7. Let $a \in S_{L, \varrho, \varrho^{\prime}}^{\mathrm{comp}}(U)$ and fix an $h$-independent constant $T \geqslant 0$ such that $e^{-t H_{p}}(\operatorname{supp} a) \subset U$ for all $t \in[0, T]$. Then,

$$
e^{i t P / h} \mathrm{Op}_{h}^{L}(a) e^{-i t P / h}=\mathrm{Op}_{h}^{L}\left(a \circ e^{t H_{p}}\right)+\mathcal{O}\left(h^{1-\varrho-\varrho^{\prime}}\right)_{L^{2} \rightarrow L^{2}} \quad \text { for } 0 \leqslant t \leqslant T .
$$

Proof. We first claim that, for each $b \in S_{L, \varrho, \varrho^{\prime}}^{\mathrm{comp}}(U)$,

$$
\left[P, \mathrm{Op}_{h}^{L}(b)\right]=-i h \mathrm{Op}_{h}^{L}\left(H_{p} b\right)+\mathcal{O}\left(h^{2-\varrho-\varrho^{\prime}}\right)_{L^{2} \rightarrow L^{2}}
$$

Using a partition of unity for $b$, we may assume that there exists a chart $\left(U^{\prime}, \varkappa, B, B^{\prime}\right)$ for $L$ such that $B$ and $B^{\prime}$ quantize $\varkappa$ near $\varkappa(\operatorname{supp} b) \times \operatorname{supp} b$ in the sense of (A.16). Then, $B^{\prime} B=I+\mathcal{O}\left(h^{\infty}\right)$ microlocally near supp $b, \sigma_{h}\left(B^{\prime} B\right)=1$ near supp $b$, and $\sigma_{h}\left(B B^{\prime}\right)=1$ near $\varkappa(\operatorname{supp} b)$. Since both $\mathrm{Op}_{h}^{L}(b)$ and $P$ are pseudolocal, we have

$$
\begin{aligned}
{\left[P, \mathrm{Op}_{h}^{L}(b)\right] } & =B^{\prime} B\left(P B^{\prime} B \mathrm{Op}_{h}^{L}(b)-\mathrm{Op}_{h}^{L}(b) B^{\prime} B P\right) B^{\prime} B+\mathcal{O}\left(h^{\infty}\right)_{L^{2} \rightarrow L^{2}} \\
& =B^{\prime}\left[B P B^{\prime}, B \mathrm{Op}_{h}^{L}(b) B^{\prime}\right] B+\mathcal{O}\left(h^{\infty}\right)_{L^{2} \rightarrow L^{2}}
\end{aligned}
$$

By (A.20), we have

$$
B \mathrm{Op}_{h}^{L}(b) B^{\prime}=\mathrm{Op}_{h}(\tilde{b})+\mathcal{O}\left(h^{\infty}\right)_{L^{2} \rightarrow L^{2}} \quad \text { for some } \tilde{b} \in S_{L_{0}, \varrho, \varrho^{\prime}}^{\mathrm{comp}}\left(T^{*} \mathbb{R}^{n}\right)
$$

with

$$
\tilde{b}=b \circ \varkappa^{-1}+\mathcal{O}\left(h^{1-\varrho}\right)_{S_{L_{0}, \varrho, \varrho^{\prime}}^{\text {comp }}\left(T^{*} \mathbb{R}^{n}\right)} \quad \text { and } \quad \operatorname{supp} \tilde{b} \subset \varkappa(\operatorname{supp} b) .
$$

Next, $B P B^{\prime} \in \Psi_{h}^{\text {comp }}\left(\mathbb{R}^{n}\right)$ and, by $\left(\right.$ A.15), $\sigma_{h}\left(B P B^{\prime}\right)=\left(p \circ \varkappa^{-1}\right) \sigma_{h}\left(B B^{\prime}\right)$ is equal to $p \circ \varkappa^{-1}$ near supp $\tilde{b}$. By (A.28), we then have $\partial_{\eta} \sigma_{h}\left(B P B^{\prime}\right)=0$ near supp $\tilde{b}$. By (A.13),

$$
\begin{aligned}
{\left[P, \mathrm{Op}_{h}^{L}(b)\right] } & =B^{\prime}\left[B P B^{\prime}, \mathrm{Op}_{h}(\tilde{b})\right] B+\mathcal{O}\left(h^{\infty}\right)_{L^{2} \rightarrow L^{2}} \\
& =-i h B^{\prime} \mathrm{Op}_{h}\left(\left\{p \circ \varkappa^{-1}, b \circ \varkappa^{-1}\right\}\right) B+\mathcal{O}\left(h^{2-\varrho-\varrho^{\prime}}\right)_{L^{2} \rightarrow L^{2}} .
\end{aligned}
$$

We have $\left\{p \circ \varkappa^{-1}, b \circ \varkappa^{-1}\right\}=\left(H_{p} b\right) \circ \varkappa^{-1} \in h^{-\varrho^{\prime}} S_{L_{0}, \varrho, \varrho^{\prime}}^{\text {comp }}\left(T^{*} \mathbb{R}^{n}\right)$. Therefore, by (A.21), the right-hand side of (A.31) is equal to $-i h \mathrm{Op}_{h}^{L}\left(H_{p} b\right)+\mathcal{O}\left(h^{2-\varrho-\varrho^{\prime}}\right)_{L^{2} \rightarrow L^{2}}$, finishing the proof of (A.30).

Now, put $a_{t}:=a \circ e^{t H_{p}}, t \in[0, T]$. By (A.28), the map $e^{t H_{p}}$ preserves the foliation $L$ on $\operatorname{supp} a$, and therefore $a_{t} \in S_{L, \varrho, \varrho^{\prime}}^{\mathrm{comp}}(U)$. Since $\partial_{t} a_{t}=H_{p} a_{t}$, by (A.30) we have

$$
\begin{aligned}
i h \partial_{t}\left(e^{-i t P / h} \mathrm{Op}_{h}^{L}\left(a_{t}\right) e^{i t P / h}\right) & =e^{-i t P / h}\left(i h \mathrm{Op}_{h}^{L}\left(\partial_{t} a_{t}\right)+\left[P, \mathrm{Op}_{h}^{L}\left(a_{t}\right)\right]\right) e^{i t P / h} \\
& =\mathcal{O}\left(h^{2-\varrho-\varrho^{\prime}}\right)_{L^{2} \rightarrow L^{2}}
\end{aligned}
$$

for $0 \leqslant t \leqslant T$. Integrating this from 0 to $t$, we get (A.29), finishing the proof. 
We now restrict ourselves to the case when $M$ is a hyperbolic surface, $U=T^{*} M \backslash\{0\}$, and $L \in\left\{L_{u}, L_{s}\right\}$ with $L_{u}$ and $L_{s}$ defined in (2.6). Let $\varphi_{t}$ be the homogeneous geodesic flow, $P \in \Psi_{h}^{\text {comp }}(M)$ be defined in $(2.11)$, and $U(t)=e^{-i t P / h}$ as in (2.13). The following statement is a version of Egorov's theorem for times up to $\varrho \log (1 / h)$, assuming that the propagated operator lies in the standard calculus $\Psi_{h}^{\text {comp }}$.

Lemma A.8. Assume that $a \in C_{0}^{\infty}\left(\left\{\frac{1}{4}<|\xi|_{g}<4\right\}\right)$ is $h$-independent. Then we have, uniformly in $t \in[0, \varrho \log (1 / h)]$,

$$
\begin{aligned}
& U(-t) \mathrm{Op}_{h}(a) U(t)=\mathrm{Op}_{h}^{L_{s}}\left(a \circ \varphi_{t}\right)+\mathcal{O}\left(h^{1-\varrho} \log (1 / h)\right)_{L^{2} \rightarrow L^{2}}, \\
& U(t) \mathrm{Op}_{h}(a) U(-t)=\mathrm{Op}_{h}^{L_{u}}\left(a \circ \varphi_{-t}\right)+\mathcal{O}\left(h^{1-\varrho} \log (1 / h)\right)_{L^{2} \rightarrow L^{2}} .
\end{aligned}
$$

Here, $a \circ \varphi_{t} \in S_{L_{s}, \varrho, 0}^{\mathrm{comp}}\left(T^{*} M \backslash\{0\}\right)$ and $a \circ \varphi_{-t} \in S_{L_{u}, \varrho, 0}^{\mathrm{comp}}\left(T^{*} M \backslash\{0\}\right)$ by (2.19) and (2.20).

Proof. We prove (A.32), with (A.33) proved similarly (replacing $P$ by $-P$ ). By property $(2)$ in $\S$ A.4, we may replace $\mathrm{Op}_{h}(a)$ by $\mathrm{Op}_{h}^{L_{s}}(a)$ with an $\mathcal{O}(h)_{L^{2} \rightarrow L^{2}}$ error.

We write $t=N s$, where $0 \leqslant s \leqslant 2$ and $N \in \mathbb{N}_{0}, N \leqslant \log (1 / h)$. Then,

$$
\begin{aligned}
& U(-t) \mathrm{Op}_{h}^{L_{s}}(a) U(t)-\mathrm{Op}_{h}^{L_{s}}\left(a \circ \varphi_{t}\right) \\
& \quad=\sum_{j=0}^{N-1} U(-j s)\left(U(-s) \mathrm{Op}_{h}^{L_{s}}\left(a \circ \varphi_{(N-1-j) s}\right) U(s)-\mathrm{Op}_{h}^{L_{s}}\left(a \circ \varphi_{(N-j) s}\right)\right) U(j s) .
\end{aligned}
$$

Since $U(j s)$ is unitary, it suffices to prove that, uniformly in $j=0, \ldots, N-1$,

$$
U(-s) \mathrm{Op}_{h}^{L_{s}}\left(a \circ \varphi_{(N-1-j) s}\right) U(s)-\mathrm{Op}_{h}^{L_{s}}\left(a \circ \varphi_{(N-j) s}\right)=\mathcal{O}\left(h^{1-\varrho}\right)_{L^{2} \rightarrow L^{2}} .
$$

Now, (A.34) follows from Lemma A.7 applied to $a \circ \varphi_{(N-1-j) s} \in S_{L_{s}, \varrho, 0}^{\text {comp }}\left(T^{*} M \backslash\{0\}\right)$. Here, $\varphi_{t}=e^{t H_{\sigma_{h}(P)}}$ on $\left\{\frac{1}{4}<|\xi|_{g}<4\right\}$, by $(2.12)$.

Acknowledgements. The authors would like to thank Jeffrey Galkowski and Maciej Zworski for several useful discussions and two anonymous referees for numerous comments used to improve the manuscript. This research was conducted during the period S.D. served as a Clay Research Fellow and L. J. as a Visiting Assistant Professor in Purdue University. 


\section{References}

[A] Anantharaman, N., Entropy and the localization of eigenfunctions. Ann. of Math., 168 (2008), 435-475.

[AN] Anantharaman, N. \& Nonnenmacher, S., Half-delocalization of eigenfunctions for the Laplacian on an Anosov manifold. Ann. Inst. Fourier (Grenoble), 57 (2007), $2465-2523$.

[AS] Anantharaman, N. \& Silberman, L., A Haar component for quantum limits on locally symmetric spaces. Israel J. Math., 195 (2013), 393-447.

[BD] Bourgain, J. \& Dyatlov, S., Spectral gaps without the pressure condition. Ann. of Math., 187 (2018), 825-867.

[B] Brooks, S., Logarithmic-scale quasimodes that do not equidistribute. Int. Math. Res. Not. IMRN, 22 (2015), 11934-11960.

[C] Coudene, Y., A short proof of the unique ergodicity of horocyclic flows, in Ergodic Theory, Contemp. Math., 485, pp. 85-89. Amer. Math. Soc., Providence, RI, 2009.

[DS] Dimassi, M. \& SJöstrand, J., Spectral Asymptotics in the Semi-Classical Limit. London Math. Soc. Lecture Note Ser., 268. Cambridge Univ. Press, Cambridge, 1999.

[D] Donnelly, H., Quantum unique ergodicity. Proc. Amer. Math. Soc., 131 (2003), 29452951.

[DFG] Dyatlov, S., Faure, F. \& Guillarmou, C., Power spectrum of the geodesic flow on hyperbolic manifolds. Anal. PDE, 8 (2015), 923-1000.

[DZa] Dyatlov, S. \& ZAhl, J., Spectral gaps, additive energy, and a fractal uncertainty principle. Geom. Funct. Anal., 26 (2016), 1011-1094.

[DZw] Dyatlov, S. \& Zworski, M., Mathematical theory of scattering resonances. Book in progress. Available at http://math.mit.edu/ dyatlov/res/.

[EN] Eswarathasan, S. \& Nonnenmacher, S., Strong scarring of logarithmic quasimodes. Ann. Inst. Fourier (Grenoble), 67 (2017), 2307-2347.

[ES] Eswarathasan, S. \& Silberman, L., Scarring of quasimodes on hyperbolic manifolds. Nonlinearity, 31 (2018), 1-29.

[FNB] Faure, F., Nonnenmacher, S. \& De Bièvre, S., Scarred eigenstates for quantum cat maps of minimal periods. Comm. Math. Phys., 239 (2003), 449-492.

[F] Furstenberg, H., The unique ergodicity of the horocycle flow, in Recent Advances in Topological Dynamics (New Haven, CT, 1972), Lecture Notes in Math., 318, pp. 95-115. Springer, Berlin-Heidelberg, 1973.

[H] Hassell, A., Ergodic billiards that are not quantum unique ergodic. Ann. of Math., 171 (2010), 605-619.

[HMR] Helffer, B., Martinez, A. \& Robert, D., Ergodicité et limite semi-classique. Comm. Math. Phys., 109 (1987), 313-326.

[HM] Hubbard, J.H. \& Miller, R. L., Equidistribution of horocyclic flows on complete hyperbolic surfaces of finite area, in In the Tradition of Ahlfors-Bers. V, Contemp. Math., 510, pp. 129-150. Amer. Math. Soc., Providence, RI, 2010.

[Ja] Jakobson, D., Quantum unique ergodicity for Eisenstein series on $\mathrm{PSL}_{2}(\mathbf{Z}) \backslash \mathrm{PSL}_{2}(\mathbf{R})$. Ann. Inst. Fourier (Grenoble), 44 (1994), 1477-1504.

[Ji1] Jin, L., Control for Schrödinger equation on hyperbolic surfaces. To appear in Math. Res. Lett.

[Ji2] - Damped wave equations on compact hyperbolic surfaces. Preprint, 2017. arXiv: 1712.02692 [math. AP].

[L] Lindenstrauss, E., Invariant measures and arithmetic quantum unique ergodicity. Ann. of Math., 163 (2006), 165-219. 
[LM] Logunov, A. \& Malinnikova, E., Quantitative propagation of smallness for solutions of elliptic equations. Preprint, 2017. arXiv:1711.10076 [math.AP].

[LS] Luo, W.Z. \& SARnak, P., Quantum ergodicity of eigenfunctions on $\mathrm{PSL}_{2}(\mathbf{Z}) \backslash \mathbf{H}^{2}$. Inst. Hautes Études Sci. Publ. Math., (1995), 207-237.

[Mc] Marcus, B., Unique ergodicity of the horocycle flow: variable negative curvature case. Israel J. Math., 21 (1975), 133-144.

[Mk] Marklof, J., Arithmetic quantum chaos. Encyclopedia Math. Phys., 1 (2006), 212-220.

[Ra] Ratner, M., Raghunathan's conjectures for SL(2, R). Israel J. Math., 80 (1992), 1-31.

[Ri2] Rivière, G., Entropy of semiclassical measures for nonpositively curved surfaces. Ann. Henri Poincaré, 11 (2010), 1085-1116.

[Ri1] - Entropy of semiclassical measures in dimension 2. Duke Math. J., 155 (2010), 271336.

[RS] RUdnick, Z. \& SARnak, P., The behaviour of eigenstates of arithmetic hyperbolic manifolds. Comm. Math. Phys., 161 (1994), 195-213.

[Sa] Sarnak, P., Recent progress on the quantum unique ergodicity conjecture. Bull. Amer. Math. Soc., 48 (2011), 211-228.

[Sh] Shnirel'man, A.I., Ergodic properties of eigenfunctions. Uspekhi Mat. Nauk, 29 (1974), 181-182 (Russian).

[So] Soundararajan, K., Quantum unique ergodicity for $\mathrm{SL}_{2}(\mathbf{Z}) \backslash$ H. Ann. of Math., 172 (2010), 1529-1538.

[CdV] Colin de Verdière, Y., Ergodicité et fonctions propres du laplacien. Comm. Math. Phys., 102 (1985), 497-502.

[Ze1] Zelditch, S., Uniform distribution of eigenfunctions on compact hyperbolic surfaces. Duke Math. J., 55 (1987), 919-941.

[Ze2] - Recent developments in mathematical quantum chaos, in Current Developments in Mathematics, 2009, pp. 115-204. Int. Press, Somerville, MA, 2010.

[ZZ] Zelditch, S. \& Zworski, M., Ergodicity of eigenfunctions for ergodic billiards. Comm. Math. Phys., 175 (1996), 673-682.

[Zw1] Zworski, M., Semiclassical Analysis. Graduate Studies in Mathematics, 138. Amer. Math. Soc., Providence, RI, 2012.

[Zw2] - Mathematical study of scattering resonances. Bull. Math. Sci., 7 (2017), 1-85.

\section{Semyon Dyatlov}

Department of Mathematics

Massachusetts Institute of Technology

77 Massachusetts Avenue

Cambridge, MA 02139

U.S.A.

and

Department of Mathematics

University of California, Berkeley

Berkeley, CA 94720

U.S.A.

dyatlov@math . berkeley .edu

Received May 26, 2017
LONG JIN

Yau Mathematical Sciences Center

Tsinghua University

Beijing

China

ljin@math.tsinghua.edu.cn

and

Department of Mathematics

Purdue University

150 N. University St.

West Lafayette, IN 47907

U.S.A. 